\title{
Blocking immunoinhibitory receptor LILRB2 reprograms tumor-associated myeloid cells and promotes antitumor immunity
}

\author{
Hui-Ming Chen,,$^{1,2}$ William van der Touw, ${ }^{3}$ Yuan Shuo Wang, ${ }^{3}$ Kyeongah Kang, ${ }^{1,2}$ Sunny Mai, ${ }^{3}$ Jilu Zhang, ${ }^{1,2}$ \\ Dayanira Alsina-Beauchamp, ${ }^{3}$ James A. Duty, ${ }^{4}$ Sathish Kumar Mungamuri, ${ }^{3}$ Bin Zhang, ${ }^{5}$ Thomas Moran, ${ }^{4}$ Richard Flavell, ${ }^{6}$ \\ Stuart Aaronson, ${ }^{3}$ Hong-Ming Hu, ${ }^{7}$ Hisashi Arase, ${ }^{8}$ Suresh Ramanathan, ${ }^{9}$ Raja Flores, ${ }^{9,10}$ Ping-Ying Pan, ${ }^{1,2}$ and Shu-Hsia Chen ${ }^{1,2}$ \\ ${ }^{1}$ Immunotherapy Research Center, and ${ }^{2}$ Cancer Center, Houston Methodist Research Institute, Houston, Texas, USA. ${ }^{3}$ Department of Oncological Sciences, ${ }^{4}$ Department of Microbiology, and ${ }^{5}$ Department of \\ Genetics and Genomic Sciences, Icahn School of Medicine at Mount Sinai, New York, New York, USA. ${ }^{6}$ Department of Immunobiology, Howard Hughes Medical Institute, Yale School of Medicine, New Haven, \\ Connecticut, USA. ' Laboratory of Cancer Immunobiology, Earle A. Chiles Research Institute, Providence Portland Medical Center, Portland, Oregon, USA. ${ }^{8}$ Department of Immunochemistry, Research Institute \\ for Microbial Diseases, Osaka University, Suita, Osaka, Japan. ${ }^{9}$ Department of Thoracic Surgery, Mount Sinai Hospital, New York, New York, USA. ${ }^{10}$ Department of General Surgery, Icahn School of Medicine at \\ Mount Sinai, New York, New York, USA.
}

\begin{abstract}
Tumor-associated myeloid cells maintain immunosuppressive microenvironments within tumors. Identification of myeloidspecific receptors to modulate tumor-associated macrophage and myeloid-derived suppressor cell (MDSC) functions remains challenging. The leukocyte immunoglobulin-like receptor B (LILRB) family members are negative regulators of myeloid cell activation. We investigated how LILRB targeting could modulate tumor-associated myeloid cell function. LILRB2 antagonism inhibited receptor-mediated activation of SHP1/2 and enhanced proinflammatory responses. LILRB2 antagonism also inhibited AKT and STAT6 activation in the presence of M-CSF and IL-4. Transcriptome analysis revealed that LILRB2 antagonism altered genes involved in cell cytoskeleton remodeling, lipid/cholesterol metabolism, and endosomal sorting pathways, as well as changed differentiation gene networks associated with inflammatory myeloid cells as opposed to their alternatively activated phenotype. LILRB2 blockade effectively suppressed granulocytic MDSC and Treg infiltration and significantly promoted in vivo antitumor effects of T cell immune checkpoint inhibitors. Furthermore, LILRB2 blockade polarized tumor-infiltrating myeloid cells from non-small cell lung carcinoma tumor tissues toward an inflammatory phenotype. Our studies suggest that LILRB2 can potentially act as a myeloid immune checkpoint by reprogramming tumorassociated myeloid cells and provoking antitumor immunity.
\end{abstract}

\section{Introduction}

Macrophages not only mediate inflammation during infection but also suppress immunity during inflammation resolution (1) and promote immune escape within tumor microenvironments (2). The functional plasticity of macrophage phenotypes is largely influenced by the surrounding milieu. Viruses and bacteria activate Toll-like receptor (TLR) signaling to drive NF- $\kappa \mathrm{B}$ and favor macrophage release of iNOS, TNF- $\alpha$, and IL-12 to support Th1 immunity, while Th1-associated IFN- $\gamma$ activates STAT1 to support effector $\mathrm{T}$ cell responses. Conversely, humoral cytokines IL-4/ IL-13 and IL-10 secreted from tumor cells stimulate macrophages to release IL-10, TGF- $\beta$, and arginase 1 . Simultaneous activation of STAT6, STAT3, and matrix proteases further supports tissue remodeling and Th2 immunity (3). Whether, and how, to target maturation pathways to control the plasticity of myeloid cell dif-

Authorship note: HMC and WVDT are co-first authors

Conflict of interest: The authors have declared that no conflict of interest exists. License: Copyright 2018, American Society for Clinical Investigation.

Submitted: September 19, 2017; Accepted: September 4, 2018

Reference information: J Clin Invest. 2018;128(12):5647-5662.

https://doi.org/10.1172/JCI97570. ferentiation and macrophage phenotypes remains a challenge for current cancer immunotherapies.

Among the myeloid receptors, paired immunoglobulin-like receptor B (PIR-B) is the sole mouse receptor ortholog of the human leukocyte immunoglobulin-like receptor B (LILRB) family (also known as ILT, LIR, and CD85) $(4,5)$. In B cells, Pirb deficiency results in increased $\mathrm{B}$ cell receptor signaling and hyperactivity (6). Pirb-deficient macrophages similarly have increased proinflammatory cytokine release and exacerbated autoimmune diseases (7). PIR-B homeostatically suppresses immune activation by binding MHC-I in cis and trans $(8,9)$. SHP1/2 phosphatases constitutively bind to the cytoplasmic domain of PIR-B and are hypothesized to be regulatory at steady state $(10,11)$. Our previous study demonstrated that PIR-B is a key regulator for maintaining the M2-like phenotype of tumor-infiltrating myeloid-derived suppressor cells (MDSCs) (12). TLR and IFN- $\gamma$ signaling was magnified in Pirb-deficient MDSCs, while IL-4/IL-13 and IL-10 were suppressed. Mice with Pirb deficiency had reduced tumor burdens, enhanced antitumor responses, decreased Treg activation, and an infiltrating macrophage profile that resembled M1-like classical activation (12). Human LILRBs, like mouse PIR-B, bear immunoreceptor tyrosine-based inhibitory motifs that can atten- 
uate signaling cascades generated from the cross-link-dependent activation of receptors bearing immunoreceptor tyrosine-based activating motifs (13). However, less is known about how LILRBs regulate human myeloid cells and macrophage activation, largely because of a lack of conservation between humans and mice, with multiple LILRB family members in humans instead of one PIR-B.

Expression of Lilrb1-Lilrb5 is enriched in myeloid cell populations and appears to be primate-specific (14-16). LILRB3 and LILRB4 are orphan receptors $(17,18)$, and LILRB5 reportedly binds $\beta_{2}$-microglobulin-free heavy chains of HLA-B27 (19). LIL$\mathrm{RB} 1$ and LILRB2 are the best-characterized receptors, as both bind to classical and nonclassical HLA class I $(17,20)$ with a low binding affinity $\left(K_{\mathrm{D}}=\sim 14-45 \mu \mathrm{M}\right.$ for LILRB2) as well as to members of the angiopoietin-like protein family $(21,22)$. Furthermore, LILRB1 and LILRB2 may compete with CD8 for HLA-I binding to potentially modulate $\mathrm{CD}^{+} \mathrm{T}$ cell responses (22). Activation of LILRB2 by viral expression of an HLA-B variant can promote myeloid cell tolerance and downregulate maturation and costimulatory molecule expression of dendritic cells (DCs) (23). Several studies have shown that HLA-G-LILRB1/2 engagement increases IL-4 and IL-13, suppresses proinflammatory cytokine release, and promotes secretion of IL-10 and TGF- $\beta$ regulatory cytokines (24). Lower levels of LILRB expression on DCs are correlated with enhanced antigen-presenting cell function (25). LILRB2 agonism has been shown to block DC Ca ${ }^{2+}$ flux (17) and plays a crucial role in the tolerization of DCs (26). Therefore, we hypothesized that targeting LILRB2 by antagonizing cell surface receptor-mediated activation may potentially alter the function of myeloid cells with immunosuppressive activities, i.e., MDSCs or tumor-associated macrophages (TAMs), toward a classically activated inflammatory macrophage phenotype.

The present study was designed to investigate whether LILRB2 is a valid and sufficient target for modulating myeloid cell functions. We generated LILRB2-specific monoclonal antibodies and found that a subset of LILRB2 antagonism altered AKT-dependent maturation of macrophages in response to macrophage colony-stimulating factor (M-CSF), and enhanced NF- $\kappa$ B and STAT1 activation in response to LPS/IFN- $\gamma$ stimuli. LILRB2 antagonism also rendered macrophages resistant to humoral cytokine-dependent STAT 6 activation by IL-4, relieved the suppressive effect of macrophages on $\mathrm{T}$ cell proliferation, and reprogrammed human macrophages from A549 lung tumor models and primary human non-small cell lung carcinoma. Furthermore, LILRB2 blockade changed the tumor microenvironment and promoted antitumor immunity when used in conjunction with anti-PD-L1.

Our findings suggest that human LILRB2 is a critical homeostatic surface regulator for myeloid cell maturation with great therapeutic value as a promising myeloid immune checkpoint target specifically aimed at myeloid cell functional determination. Blockade of LILRB2 can be used to reprogram TAMs to improve the therapeutic outcome of cancer immune therapies through modulation of the tumor microenvironment.

\section{Results}

A subset of LILRB2 antibodies enhance activation of human monocytes. In order to investigate the biological significance of the pres- cence of LILRB2 on human macrophages, we developed anti-LILRB2 antibodies by immunizing mice with a Lilrb2 cDNA-encoding plasmid followed by boosting with LILRB2 vesicles or proteins. We screened hybridoma supernatants for LILRB binding by flow cytometry followed by peripheral blood mononuclear cell-based (PBMC-based) functional assays to assess whether clones could amplify monocyte activation. Several antibody clones could enhance CD86 and TNF- $\alpha$ levels in the presence of lipopolysaccharide (LPS) across multiple PBMC donors (Figure 1, A and B). Because members of the LILRB family share a high degree of homology, we tested for potential cross-reactivity by generating cell lines stably transduced with each receptor's extracellular domain (Supplemental Figure 1A; supplemental material available online with this article; https://doi.org/10.1172/JCI97570DS1). Cross-reactivity to LILRA1 was included since this receptor shares about $80 \%$ homology with the LILRB2 extracellular domain. FACS staining demonstrated that LILRB2 antibodies did not cross-react with related family members (Figure 1C). Staining of PBMCs was also restricted to the $\mathrm{CD}^{+} 3^{+}$myeloid subset, specifically staining $\mathrm{CD} 14^{+} \mathrm{CD} 16^{\text {hi }}$ and $\mathrm{CD} 14^{+} \mathrm{CD} 16^{\text {lo }}$ monocyte populations (Supplemental Figure 1B). We identified LILRB2-specific antibodies that enhanced monocyte inflammatory potential in response to a low dose of LPS stimulus. We then determined the binding affinity of anti-LILRB2 against a THP1 human monocytic cell line that stably expresses the LILRB2 receptor (Figure 1D). Biolayer interferometry is an optical technique that measures changes in molecule interactions on an immobilized probe. Using this approach, we measured the association and dissociation of immobilized anti-LILRB2 with LILRB2-His monomers at titrated concentrations (Figure 1E). Dissociation of the complex was minimal at all LILRB2-His concentrations tested, and affinities were calculated in the range of 1.8-3.8 $\mathrm{nM}$ and were approximately 1,000-fold stronger than endogenous HLA ligand binding $\left(K_{\mathrm{D}}=\sim 14-45 \mu \mathrm{M}\right.$; ref. 22$)$.

LILRB2 antagonism alters M-CSF-dependent maturation of macrophages. Because LILRB2 antagonists amplified monocyte activation in response to LPS, we investigated how LILRB2 blockade affects macrophage maturation. Studies in human monocyte-derived macrophages have demonstrated different maturation phenotypes resulting from inflammatory cues $(27,28)$. We generated immature macrophages $\mathrm{M}(-)$ by treating $\mathrm{CD} 33^{+}$ monocytes from PBMCs of healthy donors with M-CSF for 5-7 days. While macrophages cultured in the presence of control Ig appeared elongated and loosely adherent, monocytes cultured in the presence of anti-LILRB2 appeared rounder and tightly adherent (Figure 2A). Others have reported the positive effect of $\mathrm{M}-\mathrm{CSF}$ and IL-10 on the spindle-like morphology and function of M-CSFderived human macrophages in vitro $(29,30)$. These observations suggest that LILRB2 antagonism may be interfering with typical M-CSF-dependent maturation. We observed that both CD14 and CD163 expression were diminished in response to anti-LILRB2 across all human donors tested (Figure 2, B and C). CD14 has been shown to be upregulated by M-CSF (27) and CD163 and is a scavenger receptor whose cell surface expression is correlated with antiinflammatory responses and is an indicator of poor prognosis in a variety of cancers $(31,32)$. CD163 was also enhanced in the presence of M-CSF, IL-6, and IL-10, and in response to glucocorticoids (33). To determine whether macrophages display a differ- 
A

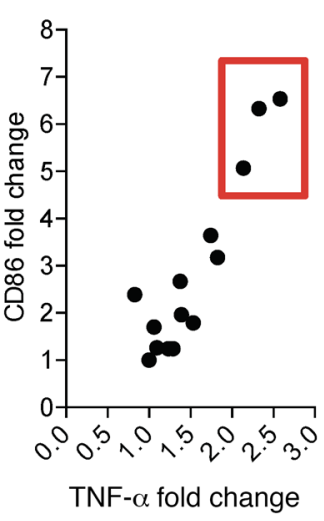

D

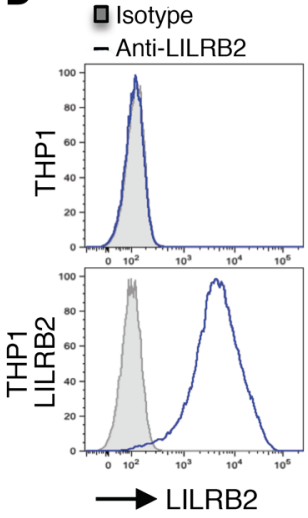

B

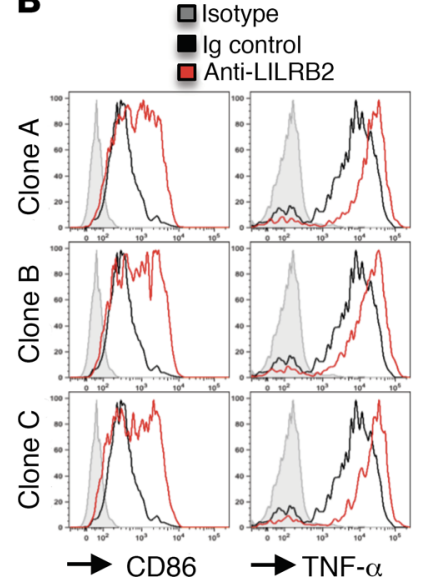

E

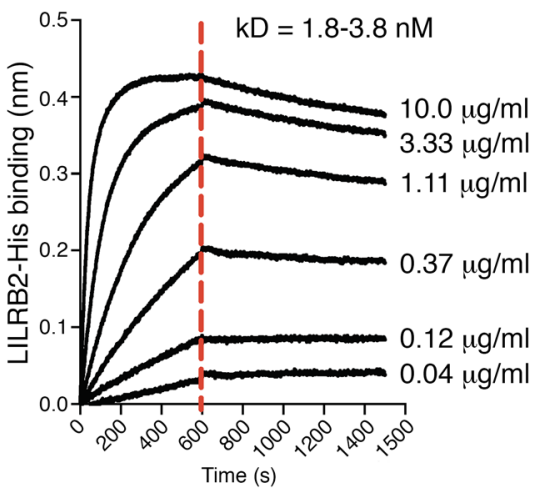

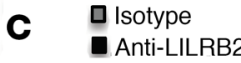

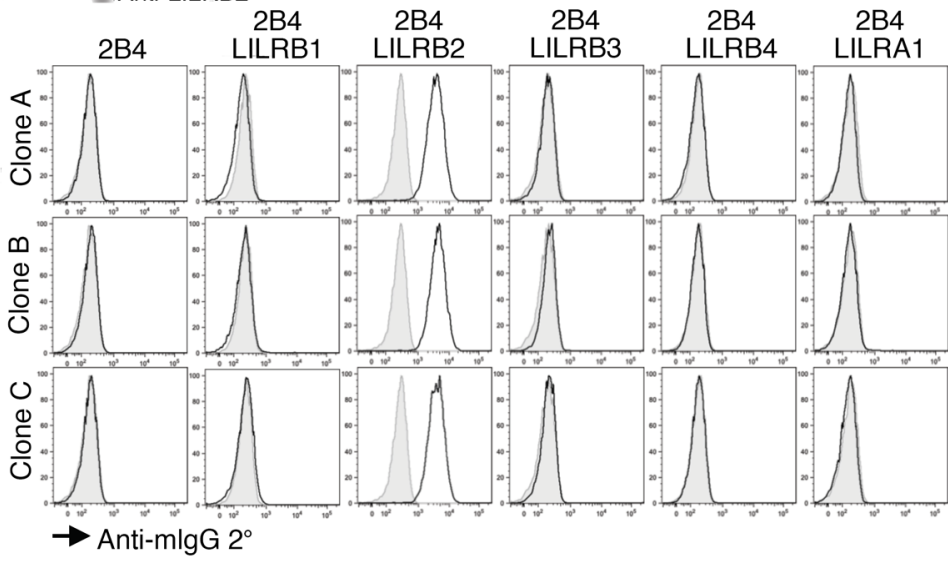

Figure 1. Anti-LILRB2 antibodies enhance LPS response in primary human monocytes. (A) Fold change of TNF- $\alpha$ and CD86 mean fluorescence intensity (MFI) levels relative to IgG-treated samples. PBMCs were cultured for 48 hours with LILRB2-reactive hybridoma supernatant followed by 6 hours of LPS stimulation $(50 \mathrm{ng} / \mathrm{ml})$ in the presence of brefeldin A. MFI values represent cells gated on $\mathrm{CD}^{+} 3^{+}$monocytes. (B) Raw CD86 and TNF- $\alpha$ data of highlighted clones (red line) from $\mathbf{A}$. Data are overlaid on Ig control-treated PBMC data (black line). (C) LILRB and LILRA1 receptor recognition of highlighted clones in A. Antibody binding was detected using goat polyclonal anti-mouse IgG secondary antibody. (D) Expression of LILRB2 on THP1 parental cells and LILRB2 retroviral transduced cells. (E) Biolayer interferometry data testing LILRB2-His association with ( $t=1-600$ seconds) and dissociation from ( $t=600-1,450$ seconds) immobilized anti-LILRB2 $(10 \mu \mathrm{g} / \mathrm{ml})$. Concentrations of LILRB2-His and calculated anti-LILRB2 affinity (clone A) are shown. ential response to LPS stimulation as was shown for monocytes, we stimulated immature macrophages overnight with LPS and measured TNF- $\alpha$ and IL-10 cytokine levels (Figure 2D). We consistently observed increased TNF- $\alpha$ and inhibited IL-10 secretion across multiple donors. No detectable TNF- $\alpha$ could be observed in macrophage cultures before LPS stimulation (data not shown), demonstrating that the increase of TNF- $\alpha$ was specific to antibody functional activity.

IRF5 is induced by type I IFN and promotes inflammatory macrophage polarization (34), which is inhibited by IRF4. IRF4 is associated with alternative macrophage maturation, is induced by IL-4, and negatively regulates TLR signaling and proinflammatory cytokines $(35,36)$. IRF3 is activated by TLR signaling and induces type I IFN/STAT1 signaling cascades (37), whereas IRF7 is reported to participate in the inflammatory microglial polarization switch (38). We observed that anti-LILRB2 upregulated IRF3, IRF5, and IRF7 but downregulated IRF4 during differentiation of immature macrophages (Figure 2E), indicating that anti-LILRB2 potentially favors classical and inflammatory macrophage polarization. To rule out any contaminating endotoxin in LILRB antibody purification, we ensured that all endotoxin levels were less than $0.005 \mathrm{EU} / \mu \mathrm{g}$ in all antibody batches.

To explore the effect of anti-LILRB2 on macrophage maturation pathways, we generated mature macrophages from M-CSFcultured M(-) followed by 24-hour stimulation with LPS or IL-4 to generate $\mathrm{M}(\mathrm{LPS})$ and $\mathrm{M}(\mathrm{IL}-4)$, respectively. Compared with $\mathrm{M}(-)$ and $\mathrm{M}(\mathrm{IL}-4), \mathrm{M}(\mathrm{LPS})$ had markedly increased expression of PD-L1, consistent with literature reporting PD-L1 upregulation in response to TLR agonists (39). Surprisingly, we found that anti-LILRB2-treated M(LPS) significantly inhibited the induced expression of PD-L1 (Figure 2, F and G). PD-L1 expression on M(IL-4) was negligible. These data suggest that LILRB2 antagonism may help prevent PD-L1-dependent suppression of effector $\mathrm{T}$ cells in inflammatory environments. We next investigated how anti-LILRB2 could affect macrophage maturation in response to the humoral cytokine IL-4. We focused on macrophage DC-SIGN (CD209), since its expression is in part IL-4-dependent (32), and it has been reported to be a well-accepted marker of $\mathrm{M}(\mathrm{IL}-4)$ macrophage maturation and immune tolerance $(27,40)$. We found that DC-SIGN was induced at low levels in $\mathrm{M}-\mathrm{CSF}-$ derived $\mathrm{M}(-)$ and $\mathrm{M}(\mathrm{LPS})$, as has been previously reported in regulatory settings (41). In response to IL-4 stimulation, M(IL-4) express high levels of DC-SIGN. M(IL-4) matured in the presence of anti-LILRB2 and showed significantly less induction of DC-SIGN expression, and basal expression of DC-SIGN was found in M(LPS) (Figure 2, H and I). Collectively, the results from in vitro macrophage culture supported that LILRB2 antagonism sensitizes maturation in favor of classically activated phenotype.

LILRB2 antagonism favors activation of $N F-\kappa B / S T A T 1$ and inhibition of STAT6 activation by $I L-4$. Groups describing macrophage maturation as M1-like (classically activated) versus M2-like (alternatively activated) largely characterize inflammatory phenotypes associated with NF-kB/STAT1 activation and STAT6 activation, respectively (3). We, therefore, assessed whether signaling pat- 
A

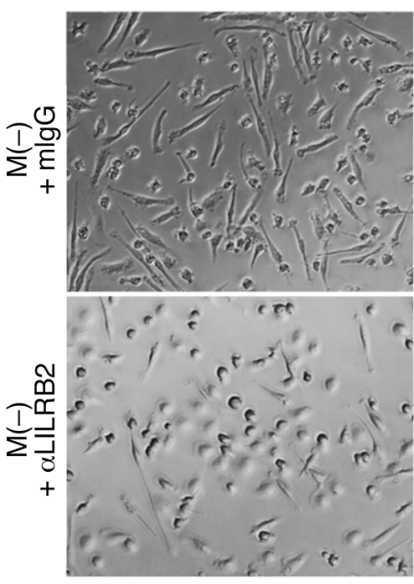

B

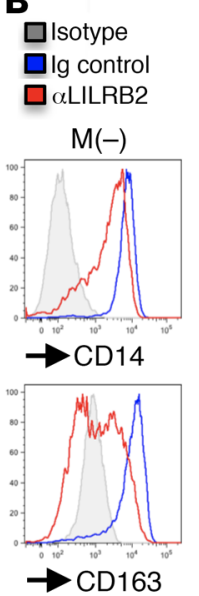

C

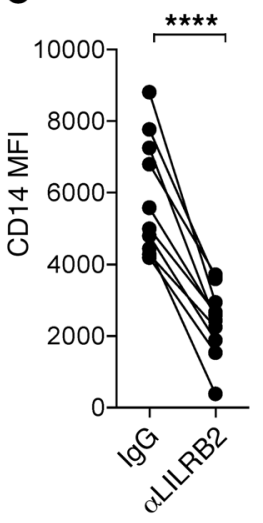

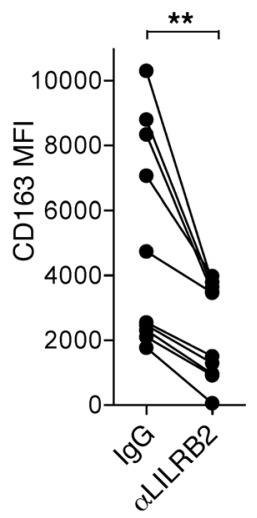

D

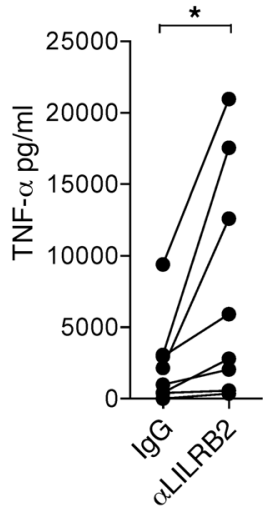

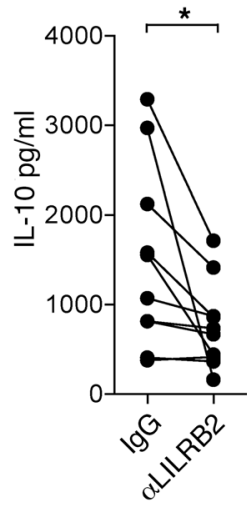

E

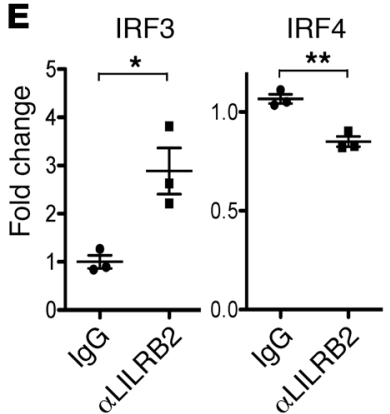

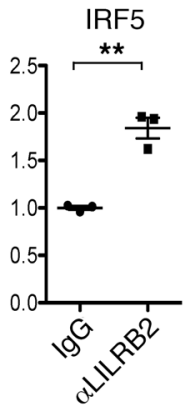

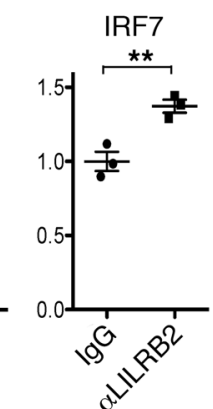

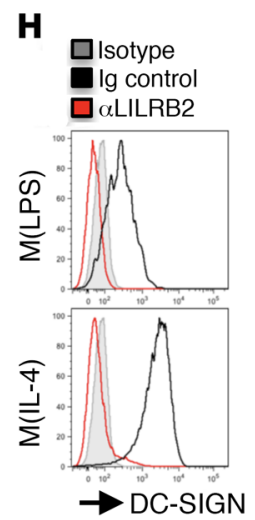

I

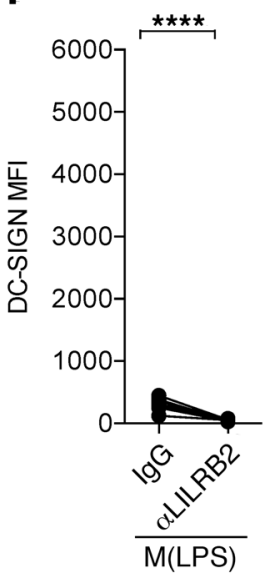

$\mathbf{F}$
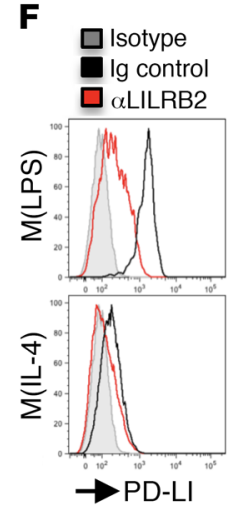

G
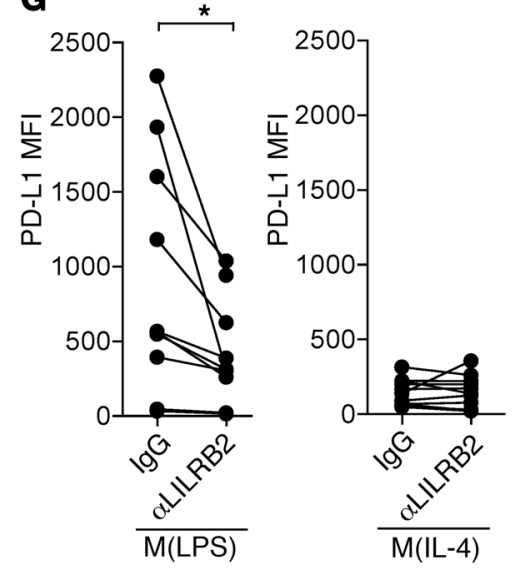

Figure 2. LILRB2 antagonism generates inflammatory macrophages in the presence of M-CSF. (A) Representative bright-field microscopy images of M-CSF $\mathrm{M}(-)$ macrophages matured in the presence of mIgG2a or anti-LILRB2 ( $\alpha$ LILRB2, 1 $\mu \mathrm{g} / \mathrm{ml}$ ). Original magnification, x20. (B) Representative levels of CD14 and CD163 expression by $\mathrm{M}(-)$ in the presence of mIgG2a (blue line) or anti-LILRB2 (red line). (C) Pooled paired MFI data from B collected from healthy donors $(n=10)$ over multiple experiments. (D) Secreted TNF- $\alpha$ and IL-10 from macrophages in $\mathbf{C}$ following 16 -hour LPS $(50 \mathrm{ng} / \mathrm{ml})$ stimulation and detection by ELISA. (E) Fold changes in IRF3, 4, 5, and 7 mRNA expression relative to the mean value in IgG-treated immature macrophages cultured in the presence of M-CSF $(50 \mathrm{ng} / \mathrm{ml})$ for 2 days. $P$ value was calculated using 2-tailed Student's $t$ test. (F) Representative PD-L1 expression in M(LPS) and M(IL-4) matured in the presence of mlgG2a (black) and anti-LILRB2 (red). (G) Pooled paired MFI data from $\mathbf{F}$ collected from healthy donors $(n=11)$ over multiple experiments. (H) Representative DC-SIGN expression of M(LPS) and M(IL-4) matured in the context of mlgG2a (black) and anti-LILRB2 (red). (I) Pooled paired MFI data from $\mathbf{H}$ collected from healthy donors $(n=11)$ over multiple experiments. $P$ value was calculated using 2 -tailed paired $t$ test. ${ }^{*} P<$ $0.05,{ }^{* *} P<0.005,{ }^{* * * *} P<0.0001$.

terns were regulated by LILRB2 antagonism. In response to LPS stimulation, anti-LILRB2-treated cells demonstrated increased NF- $\mathrm{B}, \mathrm{ERK} 1 / 2$, and $\mathrm{p} 38$ activation (Figure $3 \mathrm{~A}$ ). We found similar increases in NF- $\mathrm{B}, \mathrm{ERK} 1 / 2$, and $\mathrm{p} 38$ phosphorylation as well as STAT1 phosphorylation in response to IFN- $\gamma$ (Figure 3B). Because of reduced macrophage DC-SIGN expression in the presence of LILRB2 antagonism, we also determined whether IL-4-associated signaling was inhibited. In response to IL-4 treatment, anti-LILRB2-treated cells showed strong inhibition of STAT6 phosphorylation (Figure $3 \mathrm{C}$ ), accompanied by increased SOCS3 with no sig- nificant changes in SOCS1 expression (Figure 3D). In addition to inflammatory cytokines, M-CSF drives proliferation and survival of macrophages that depends on PI3K/AKT activation $(42,43)$, and knockout models suggest important roles of AKT in determining maturation phenotype (44). We consistently observed diminished levels of AKT activation in anti-LILRB2-treated macrophages despite the presence of exogenous M-CSF (Figure 3E). Collectively, our data suggest that LILRB2 blockade increased sensitivity to inflammatory signaling cascades by inhibiting PI3K/ AKT pathways downstream of M-CSF. 
A

\begin{tabular}{|c|c|c|c|c|c|c|c|c|}
\hline & \multicolumn{4}{|c|}{$\lg G$} & \multicolumn{4}{|c|}{$\alpha$ LILRB2 } \\
\hline LPS (min) & 0 & 5 & 10 & 30 & 0 & 5 & 10 & 30 \\
\hline JF-кB p65 & - & - & - & - & - & - & - & - \\
\hline p-ERK $1 / 2$ & 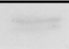 & 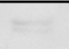 & 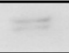 & 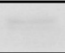 & 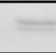 & $=$ & - & $=$ \\
\hline & & & $z$ & $3=$ & $=$ & 3 & - & - \\
\hline & & & & & & & & \\
\hline
\end{tabular}

C

\begin{tabular}{|c|c|c|c|c|c|c|c|c|}
\hline \multirow{2}{*}{ IL-4 (min) } & \multicolumn{4}{|c|}{$\lg G$} & \multicolumn{4}{|c|}{$\alpha$ LILRB2 } \\
\hline & 0 & 5 & 10 & 30 & 0 & 5 & 10 & 30 \\
\hline & & - & - & - & & - & - & - \\
\hline & & & & & & & & \\
\hline
\end{tabular}

D

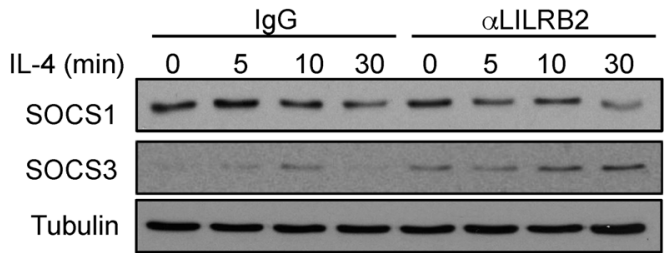

B

\begin{tabular}{|c|c|c|c|c|c|c|c|c|}
\hline \multirow[b]{2}{*}{ IFN- $\gamma(\min )$} & \multicolumn{4}{|c|}{$\lg G$} & \multicolumn{4}{|c|}{$\alpha$ LILRB2 } \\
\hline & 0 & 5 & 10 & 30 & 0 & 5 & 10 & 30 \\
\hline$p-N F-\kappa B ~ p 65$ & - & - & - & - & - & $\longrightarrow$ & - & - \\
\hline $\mathrm{p}-\mathrm{ERK} 1 / 2$ & $=$ & $=$ & $=$ & $=$ & $=$ & $=$ & $=$ & $=$ \\
\hline$p-p 38$ & & 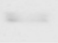 & $=$ & & & & & - \\
\hline p-STAT1 & . & - & - & - & & 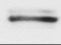 & $=$ & $=$ \\
\hline Actin & & & & & & & & - \\
\hline
\end{tabular}

$\mathbf{E}$
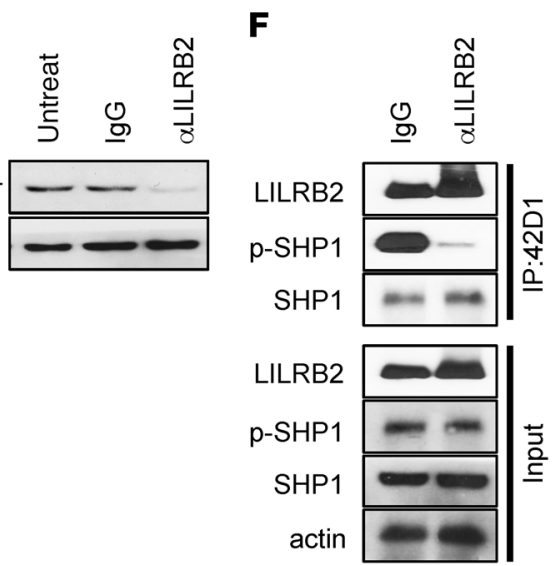

G lg control a aLILRB2

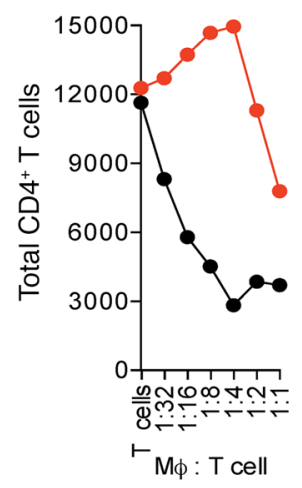

H

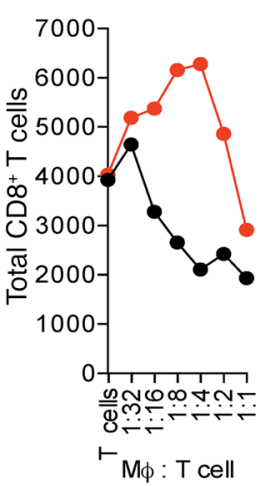

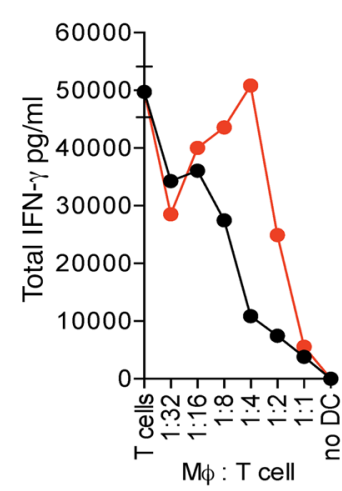

I

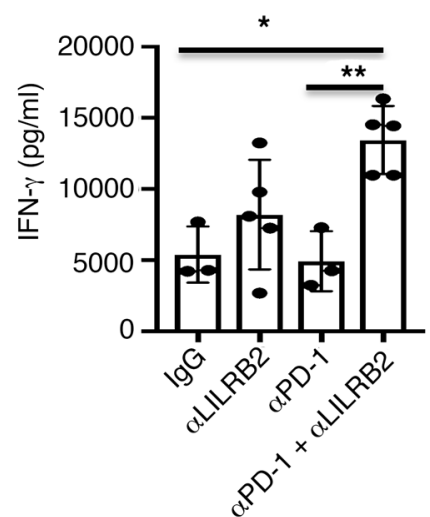

Figure 3. LILRB2 antagonism favors NF-KB/STAT1 inflammatory pathways. (A-C) THP1 LILRB2+ cells were cultured with IgC or anti-LILRB2 ( $\alpha$ LILRB2, 1 $\mu \mathrm{g} / \mathrm{ml}$ ) for 24 hours followed by acute stimulation with LPS (A), IFN- $\gamma$ (B), or IL-4 (C) for 5, 10, and 30 minutes. (A) Immunoblot of phosphorylated NF- $\mathrm{kB}$, ERK1/2, and p38 in response to LPS $(50 \mathrm{ng} / \mathrm{ml}$ ) stimulation. (B) Immunoblot of phosphorylated NF- $\mathrm{KB}, \mathrm{ERK} 1 / 2, \mathrm{p} 38$, and STAT1 in response to IFN- $\gamma$ (20 $\mathrm{ng} / \mathrm{ml}$ ) stimulation. (C) Immunoblot of phosphorylated STAT6 in response to IL-4 (20 ng/ml) stimulation. (D) Immunoblot of SOCS1 and SOCS3 in response to IL-4 $(20 \mathrm{ng} / \mathrm{ml})$ stimulation. (E) Immunoblot of phosphorylated AKT from primary M-CSF macrophages matured in the presence of IgC or anti-LILRB2. Representative data from 3 independent PBMC donors. (F) LILRB2, p-SHP1, and total SHP1 immunoblotting from IgG- and anti-LILRB2-treated macrophages. Results from anti-LILRB2 (42D1) immunoprecipitate (top) and total input lysate (bottom). (C) LILRB2 antagonism inhibits monocyte/macrophage-mediated suppression of effector T cell responses. Total CD4+ and CD8 ${ }^{+} \mathrm{T}$ cell counts determined by flow cytometry of 72 -hour mixed lymphocyte reactions (MLRs) containing mature DCs, sorted allogeneic T cells, and titrated ratios of M-CSF macrophages matured in the presence of IgC (black line) or anti-LILRB2 (red line). (H) Supernatants from MLRs in G were analyzed for secreted IFN- $\gamma$ by ELISA. (I) Total PBMCs were incubated with anti-LILRB2 (5 $\mu \mathrm{g} / \mathrm{ml})$ or lgG overnight followed by anti-PD-1 treatment $(1 \mu \mathrm{g} / \mathrm{ml})$ in the presence of OKT3 stimulation $(0.01 \mu \mathrm{g} / \mathrm{ml})$ for 3 days. Supernatants were harvested for IFN- $\gamma$ detection by ELISA. Data are from a representative experiment of 3 independent experiments and are presented as mean \pm SEM, and $P$ values were calculated using 1-way ANOVA followed by Tukey's multiple-comparisons test; ${ }^{*} P<0.05,{ }^{* *} P<0.01$.

LILRB receptors have been shown to constitutively recruit and activate SHP1 in macrophage populations (16). Coimmunoprecipitation experiments demonstrated that LILRB2-associated SHP1 phosphorylation was diminished in anti-LILRB2-treated macrophages (Figure 3F). Thus, disruption of SHP1 activation downstream of LILRB2 may explain the increased sensitization of signaling cascades.

LILRB2 antagonism inhibits myeloid-dependent suppression on effector $T$ cells. Since LILRB2 antagonism promoted inflammatory pathways supportive of Th1 adaptive immunity, we hypothesized 
A

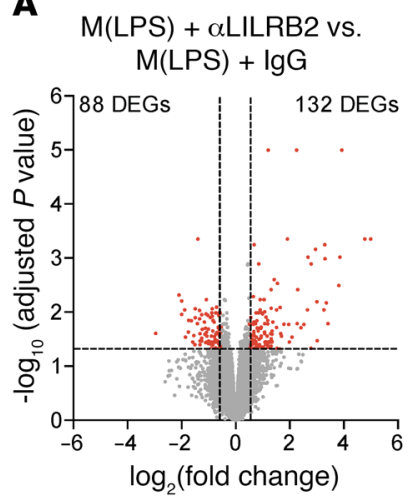

B

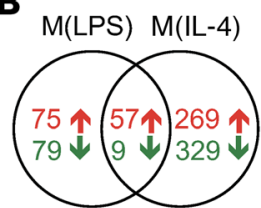

Shared LILRB2-regulated 66 DEGs

$6 \uparrow 3 \downarrow$ Immune response

$8 \uparrow 1 \Downarrow \mathrm{Lipid} /$ cholesterol homeostasis

$5 \uparrow 2 \Downarrow$ Cytoskeleton

6个 Signaling transduction regulation

4N Tissue remodeling

$2 \uparrow 1 \Downarrow$ Cell cycle, proliferation, survival

71 Cell surface molecules

$2 \uparrow \quad$ Stress response resistance

$2 \uparrow 1 \Downarrow$ Metabolism

9个 Other

$8 \uparrow 1 \Downarrow$ Unknown, NA

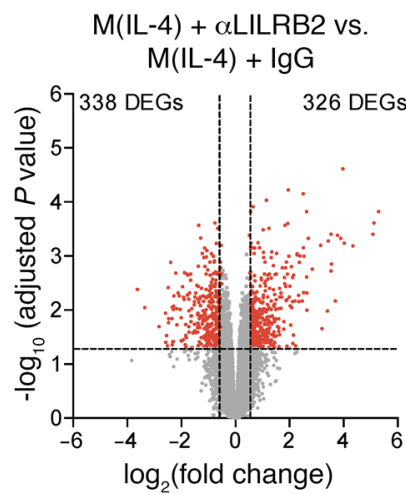

C

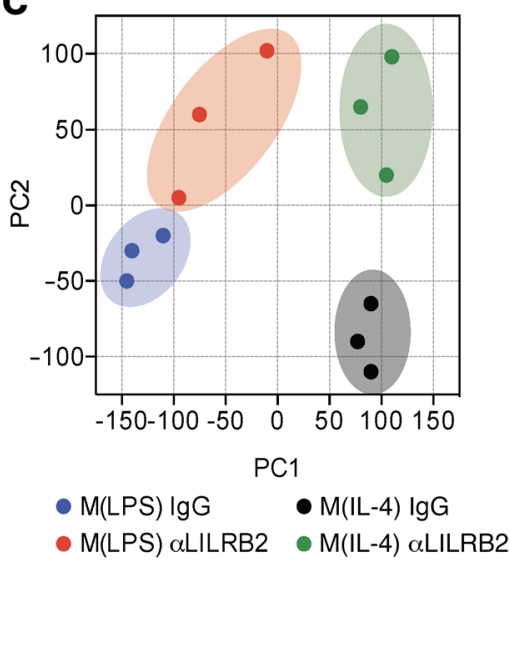

D

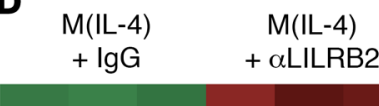

CCL2

LILRA3

PPARG

CD83

FGR

LGALS3

LAT

TNFSF14

AADACL1

C17ORF87

RIPK2

CD1B

CD300LB

CCRL2

CKLF

CD276

CSF1

SPN

SRC

TNFRSF12A

BCAP31

TREM2

FYN

SIGLEC1

TLR5

PLCL2

TLR4

IL13RA1

NLRP3

HCK

IRF9

CD1C

C2

TNFRSF1A

CYSLTR1

CD302

HLA-DRB4

HLA-DMA

CASP1

IFITM2

CSF2RA

LTC4S

FGL2

CTSC

CD163L1

HS. 197143

FCER1A

FCER2

FCGR2A

CD209

STAB1
CD14

ANGPTL4

CCL23

CCL15

COLEC12

MAF

M160

LILRB5

CCL14

CCL13

$\mathrm{CCL} 26$

CCL8

that effector $\mathrm{T}$ cell responses would be improved in the presence of anti-LILRB2-matured macrophages. Macrophages and MDSCs are known suppressors of effector $\mathrm{T}$ cell responses, using a variety of mechanisms including tryptophan catabolism by indoleamine 2,3-dioxygenase, PD-L1/L2 expression, and cytokine-dependent Treg conversion. To test the suppressive nature of anti-LILRB2matured macrophages in native adaptive $\mathrm{T}$ cell responses, we per- formed 1-way mixed lymphocyte reactions. After 3 days, clonal populations of outgrowing alloantigen-specific T cells were observed (Supplemental Figure 2, A and B). Total numbers of $\mathrm{CD}^{+}$and $\mathrm{CD} 8^{+}$ $\mathrm{T}$ cells were determined by FACS. The macrophages generated in the presence of anti-LILRB2 showed restored $\mathrm{CD} 4^{+} / \mathrm{CD}^{+} \mathrm{T}$ cell numbers (Figure $3 \mathrm{G}$ ) and IFN- $\gamma$ secretion (Figure $3 \mathrm{H}$ ), indicating that anti-LILRB2 diminished the suppressive capacity of macro- 
A

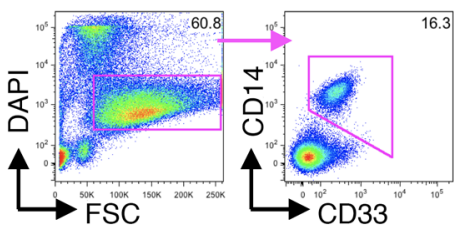

B

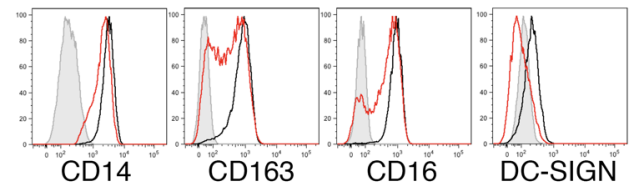

C

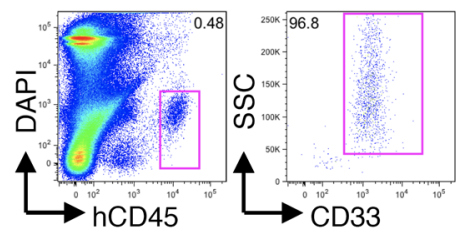

D
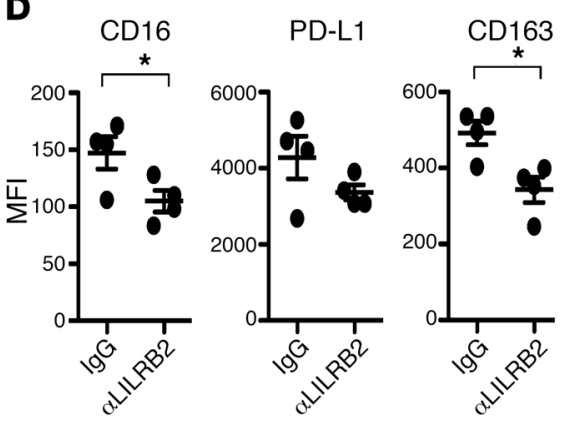

CD14

HLA-DR

E

$\mathbf{F}$

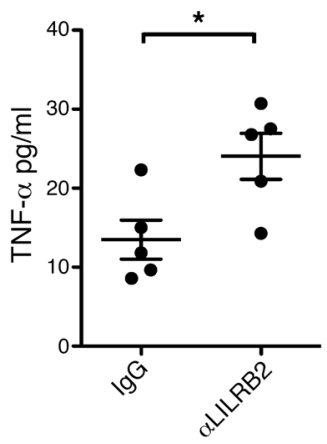

G

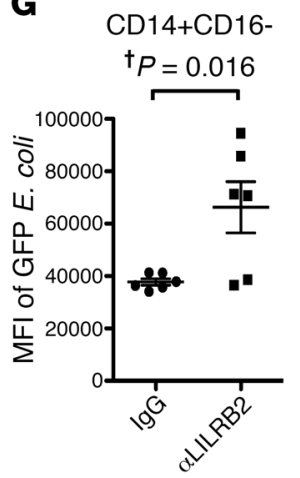

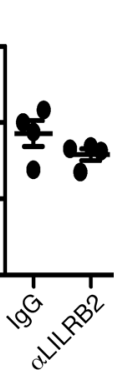
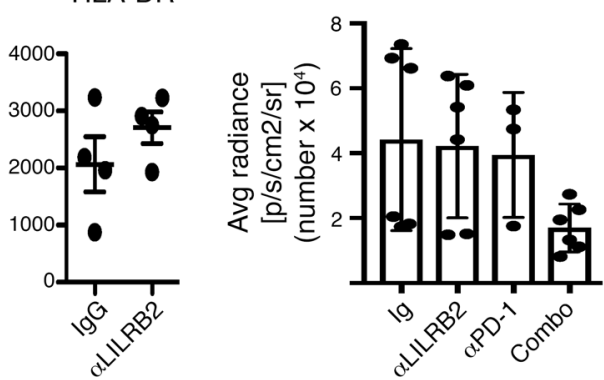

Figure 5. LILRB2 blockade reprograms lung TAM maturation in vitro and in vivo. (A) FACS gating analysis of primary human CD $33^{+}$monocytes $\left(1 \times 10^{5}\right.$ cells per well) with 5 days of coculture of A549 cells $\left(1 \times 10^{3}\right.$ cells per well). (B) CD14, CD16, CD163, and DC-SICN expression among live CD33 ${ }^{+}$CD14 ${ }^{+}$myeloid cells from A. IgG-treated cells (black line) are overlaid by anti-LILRB2-treated cells ( $\alpha$ LILRB2, red line). (C) FACS gating strategy for identifying human CD45+CD33+ macrophages from NSG-SGM3 immunodeficient mice inoculated s.C. with A549 tumor and CD33+ monocytes 12 days earlier. (D) Representative data from 1 donor are shown. Data were averaged from 4 tumor samples per group, 2 mice per group. Individual data from 5 donors are presented in Supplemental Figure 6B. Combined data from all 5 donors for CD14, HLA-DR, and CD16 are presented in Supplemental Figure 6D. Data were averaged from 4-8 tumor samples per group per donor, 2-4 mice per group per donor, 3 independent experiments. ${ }^{*} P<0.05$, by 2 -tailed Student's $t$ test. (E) HLA-A2-matched humanized NCG mice were used to assess in vivo antitumor responses by LILRB2 blockade ( $\alpha$ LILRB2) together with anti-PD-1 ( $\alpha$ PD-1) treatment in a LUC-A549 (HLA-A2+) xenograft mouse model. Statistical analysis of luciferase activity in lungs after 19 days of luciferase-expressed A549 tumor inoculation in HLA-A2+ PBMC humanized NCG mice was quantified by bioluminescence signals (Avg radiance [p/s/cm²/sr]) and is shown in the bar graph ( $n=3-6$ mice per group), using 1-way ANOVA followed by Tukey's multiple comparisons test. (F) ELISA analysis of serum TNF- $\alpha$ levels from CpG-challenged mice is shown $(n=5){ }^{*} P<0.05$, by 2-tailed Student's $t$ test. (G) The MFI of CFP-expressing E. coli in viable CD14 ${ }^{+}$CD16- and CD14 ${ }^{+}$CD16 cells in $\mathrm{CD}_{4} 5^{+} \mathrm{CD} 33^{+}$population from IgG- or anti-LILRB2-treated humanized MISTRG mice was analyzed ( $n=3$ duplicates per mouse). ${ }^{*} P<0.05$ by 2-tailed Student's $t$ test.

phages as compared with IgG treatment. In PBMC cultures stimulated with a low dose of OKT3, anti-LILRB2 significantly synergized with PD-1 blockade to enhance effector T cell secretion of IFN- $\gamma$ (Figure 3I). Altogether, we demonstrated that LILRB2 antagonism induced a macrophage phenotype that enhances adaptive Th1 effector T cell responses.

LILRB2 blockade alters $M(I L-4)$ immune, migratory, and vesicle trafficking pathways. Transcriptome studies can provide additional insight into the maturation and phenotypic changes of macrophages. Early microarray studies comparing M(LPS) and M(IL-4) populations have defined markers and pathway networks associated with M1-like versus M2-like functional phenotypes (27). Using the same experimental conditions in the context of anti-LILRB2, we performed Illumina microarray analysis to determine how LILRB2 blockade may affect transcriptional networks in M(LPS) and $\mathrm{M}(\mathrm{IL}-4)$. Comparison of M(LPS) and M(IL-4) IgG-treated transcriptomes identified 3,926 differentially expressed genes (DEGs) (Supplemental Figure 3, A and B) with significant overlap between the top 100 and those originally described by Mantovani and colleagues (27). Anti-LILRB2 versus IgG treatment of M(LPS) yielded 220 DEGs, whereas the same treatments in M(IL-4) yielded 664 DEGs (Figure 4A). The changes in 66 DEGs were conserved between anti-LILRB2-treated M(LPS) and M(IL-4) (Figure 4B). Collectively, these data demonstrate that LILRB2 antagonism results in substantially more transcriptional changes under conditions of IL-4 maturation versus LPS maturation, and the 66 con- 
served DEGs indicate that LILRB2 antagonist-specific gene alterations are independent of cytokine-induced maturation. Genes regulating immune function, lipid/cholesterol homeostasis, and cytoskeletal homeostasis were most prominent. In both M(LPS) and M(IL-4), LILRB2 blockade upregulated LILRA3 transcript, a secreted LILR family member that may further compete with other LILR family members. Anti-LILRB2 increased Ccl22, FGR, and Trem 2 transcription as well as M1-associated $S p h k 1$ transcription but decreased Siglec1, PLC decoy messenger Plcl2, and complement protein C2 transcription as well as M2-associated Klf2 transcripts. Principal component analysis of M(LPS) and M(IL-4) in response to IgG or anti-LILRB2 suggests that LILRB2 blockade generates unique macrophage phenotypes that are dissimilar to each other (Figure $4 \mathrm{C}$ ).

The finding that LILRB2 blockade has a more significant impact on M(IL-4) versus M(LPS) global transcription suggests that LILRB2 may be more important for maintaining immune homeostasis in the steady state or M2-like macrophages versus M1-like macrophages. We applied gene ontology analysis using the GOrilla platform $(45,46)$ to provide a functional interpretation of the raw data. M(LPS) DEGs were associated with G-coupled protein receptor signaling, transport, and other cellular processes. In M(IL-4), LILRB2 blockade significantly altered genes associated with immune function, as well as cell migration/motility, lipid metabolism, apoptosis/proliferation, and increased enzymatic remodeling/activity (Supplemental Figure 4, C and D); however, no clear association between LPS stimulation and immune function was noted (Supplemental Figure 4, A and B). In response to anti-LILRB2, M(IL-4) genes associated with immune function were properly regulated (Figure 4D). Interestingly, CD83, Light, Ripk2, and Tweakr transcripts implicated in enhanced adaptive immunity and costimulation were upregulated. Consistent with previous reports $(27,47)$, M1-associated genes were upregulated, including Pfkp, Sphk1, Slc31a2, Serpine1, Hsd11b, etc. (Supplemental Figure 4E). Sphingosine kinase 1 (Sphk1) is associated with obese adipose tissue macrophages from high-fat diet and ob/ob mice (48). Compared with M1-associated genes, more M2-associated genes were downregulated by anti-LILRB2, including Il13ra1, CD302, Fgl2, cathepsin C, CD163L1, Dc-sign (CD209), Maf, Ccl13, Ccl23, Stab1, and Tlr5 (Figure 4D). In M(IL-4), LILRB2 blockade suppressed MAF, an important regulator of the macrophage enhancer landscape and M2-associated gene expression (49). Other M2-associated genes were also downregulated, including Klf2/Klf4, Tgfbr2, Ms4a6a, Alox15, etc. (Supplemental Figure $4 \mathrm{E})$. The biological role of these molecules in M1/M2 differentiation remains to be elucidated. Notably, in addition to increased Lilra3 levels, M(IL-4) treated with anti-LILRB2 showed significant inhibition of Lilrb5 and Angptl4 transcript. Chemokine transcript levels were also strongly inhibited, e.g., Ccl8, Ccl26, Ccl13, Ccl14, Ccl15, and Ccl23. We also observed that LILRB2 blockade suppressed the LPS-induced dual-specificity protein tyrosine phosphatases Dusp10 (also known as MKP-5 or JNK and p38 pathwayassociated phosphatase) (50) and Dusp22 (also known as JSP-1 or JNK pathway-associated phosphatase) (51), which mediates negative feedback control of the inflammatory response.

In summary, our transcriptome-wide analyses demonstrated that LILRB2 blockade results in phenotypic changes in macro- phage maturation that include immune, as well as metabolic, sorting, and cytoskeletal changes.

LILRB2 blockade can alter tumor cell-induced macrophage maturation and promote antitumor immunity. We determined whether LILRB2 blockade could alter the macrophage phenotype in the presence of tumor-derived factors and the tumor microenvironment. Some tumor cells are sufficient to promote the autocrine production of M-CSF by monocytes in a process dependent on tumor cell-derived IL-6 (52). We hypothesized that coculture of tumor cells with human monocytes would be sufficient to generate macrophages. Gating on $\mathrm{CD} 33^{+} \mathrm{CD} 14^{+}$ cells, we reproduced the finding that A549 non-small cell lung carcinoma (NSCLC) cells were sufficient to generate macrophages whose profile resembled macrophages derived from M-CSF cultures (Figure 5A). We further observed that A549derived macrophages cultured in the presence of LILRB2 antagonist had decreased levels of CD14, CD163, CD16, and DC-SIGN (Figure 5B), consistent with our observations on M-CSF primary macrophage cultures (Figure 2). The impact of LILRB2 antagonist on Hs578T breast cancer cell-derived macrophages was similar to its impact on A549 NSCLC cells (Supplemental Figure 5, A and B). We next assessed whether A549 cells could generate primary human macrophages when coinjected s.c. in immunodeficient NSG-SGM3 mice and whether LILRB2 blockade could similarly reprogram macrophages in vivo (Supplemental Figure 6). FACS gating on human $\mathrm{CD} 45^{+} \mathrm{CD} 33^{+}$cells identified human myeloid cells from tumor single-cell suspensions (Figure $5 \mathrm{C}$ ). FACS analysis of immune markers showed that specific reductions in CD163 were reproducibly detected in 4 of 5 total donors (Supplemental Figure 6B). Furthermore, changes in other markers, including CD14, CD16, and PD-L1, were variable across donors in response to anti-LILRB2. Figure 5D shows representative data from 1 donor, while combined data from all 5 donors for CD14, HLA-DR, and CD16 are presented in Supplemental Figure 6D. CD14 and CD16 were significantly downregulated while HLA-DR was upregulated following anti-LILRB2 treatment across multiple mice and multiple human monocyte donors using paired comparative analysis (Supplemental Figure 6D). HLA-A2-matched humanized NCG mice were used for in vivo experiments in order to trace long-term antitumor responses by LILRB2 blockade together with anti-PD-1 treatment, using an A549 (HLA-A2 ${ }^{+}$) xenograft model with luciferase-expressing A549 (LUC-A549) cells. The results showed that the anti-LILRB2 and anti-PD-1 combination substantially decreased LUC-A549 tumor burden (Figure 5E). To assess the promotion of M1 phenotypes of human macrophages by anti-LILRB2 in vivo, we generated a humanized mouse model using human $\mathrm{CD}_{3} 4^{+}$cord blood stem cell-engrafted MISTRG mice to study in vivo responses of human macrophages. MISTRG mice support xenogenic human hematopoietic development due to humanized knock-in alleles (M-CSF ${ }^{\mathrm{h}}, \mathrm{IL}-3 / \mathrm{GM}-\mathrm{CSF}^{\mathrm{h}}$, and $\left.\mathrm{TPO}^{\mathrm{h}}\right)$ important for innate immune cell development that enable the full recapitulation of human myeloid development and function in the mouse. Similar human $\mathrm{CD}_{3}{ }^{+}$cell engraftment rates were observed between experimental groups (Supplemental Figure 6C). The humanized mice were treated with $\mathrm{CpG}$, and TNF- $\alpha$ concentration in serum was measured. Interestingly, approximately 2-fold higher human 
TNF- $\alpha$ levels were found in mice treated with anti-LILRB2 versus control Ig (Figure 5F). The effect of LILRB2 on phagocytosis in this humanized mouse model was tested using GFP-expressing E. coli. Anti-LILRB2 significantly increased the phagocytic activity of $\mathrm{CD} 14^{+} \mathrm{CD} 16^{-}$monocytes on GFP-expressing E. coli in comparison with the control Ig-treated group (Figure $5 G$ ).

Taken together, our data indicate that anti-LILRB2 can reprogram human macrophages in vivo to enhance antitumor responses in a xenograft human lung cancer model, a systemic response to $\mathrm{CpG}$ challenge and phagocytic activities of macrophages in humanized MISTRG mice. The overall results indicate that antagonistic LILRB2 antibody can modulate macrophage function to an M1-associated phenotype and further enhance proinflammatory responses in vivo.

LILRB2 blockade can enhance antitumor responses and decrease MDSC and Treg populations in syngeneic Lewis lung carcinoma-bearing mice. Since LILRB2 is only expressed on human, not mouse, cells and exhibits very low homology to the mouse Pirb gene, our antibody did not cross-react in the mouse system. Therefore, we used BAC DNA injection to develop LILRB2-transgenic mice. These mice highly expressed LILRB2/3 on CD11 ${ }^{+}$cells in the peripheral blood (Figure 6A), and anti-LILRB2 treatment increased the $\mathrm{CD}^{\circ} 6^{+} \mathrm{MHC}-\mathrm{II}^{+}$population (Figure 6, B and $\mathrm{C}$ ) as well as TNF- $\alpha$ and IL-10 secretion in response to LPS stimulation in vitro (Figure 6D). We evaluated whether LILRB2 blockade alone or in combination with anti-PD-L1 treatment could inhibit tumor progression and modulate the tumor microenvironment in a Lewis lung carcinoma (LLC) model. PD-L1 blockade had no effect on tumor growth, while LILRB2 blockade showed moderate antitumor effect. Blockade of both PD-L1 and LILRB2 led to significantly reduced tumor size and weight in LLC tumor-bearing mice (Figure 6E). Furthermore, the combination of anti-LILRB2 and anti-PD-L1 significantly decreased the granulocytic Ly $6 \mathrm{G}^{+}$ Ly6 $C^{\text {int }}$ MDSC population but increased monocytic Ly6 $\mathrm{G}^{-} \mathrm{Ly} \mathrm{C}^{\mathrm{hi}}$ MDSCs in tumor tissues (Figure 6F). When anti-LILRB2 was combined with anti-PD-L1 treatment, the $\mathrm{CD}^{+} \mathrm{CD} 25^{+} \mathrm{FoxP}^{+}$Treg populations in spleen and tumor-infiltrated lymphocytes were both substantially decreased (Figure 6G). Overall, LILRB2 blockade decreased MDSC and Treg populations and enhanced the therapeutic efficacy of anti-PD-L1 treatment in vivo.

Primary TAMs respond to LILRB2 blockade ex vivo. We next determined whether LILRB2 blockade could reprogram isolated primary tumor-associated macrophages (TAMs) ex vivo. Single-cell suspensions of collagenase-digested tumor tissues were used to isolate tumor-infiltrating leukocytes. By gating on DAPI-negative live cells and human CD45, we could identify infiltrating TAMs and MDSCs based on CD33, CD14, and HLA-DR gating (Figure 7A). Staining for LILRB family members revealed variable but ubiquitous expression of LILRB1, LILRB2, LILRB3, and LILRB4 among MDSC and TAM populations (Figure 7, B and C), while DCs isolated from cancer patients' tumor tissues or derived from healthy donor PBMCs expressed LILRB2 (Supplemental Figure 8, A and B). To investigate whether tumor-infiltrating myeloid populations could respond to LILRB2 blockade, we stimulated tumor-infiltrating leukocytes ex vivo for 48 hours with M-CSF in the presence or absence of IFN- $\gamma$ or anti-LILRB2. Monocytes from most patients showed enhanced TNF- $\alpha$ release with the treatment of anti-LILRB2 (Figure 7D and Supplemental Figure 7). Analyses of the cell surface markers CD163, DC-SIGN, and CD14 showed significantly diminished expression in response to LILRB2 blockade. Although a trend of decreased CD16 and PD-L1 expression was observed, it was not statistically significant (Figure 7E). These experiments were only 48 hours in duration and inclusive of total leukocyte populations found in the tumor. Nevertheless, our ex vivo data demonstrate that TAMs from patient biopsies express a wide range of LILRB family members and are responsive to LILRB2 antagonism.

\section{Discussion}

Our study demonstrates that antagonism of LILRB2 by specific monoclonal antibodies is sufficient to enhance inflammatory responses in monocytes and directly alters downstream macrophage maturation phenotypes. We observed constitutive activation of SHP1 downstream of LILRB2 that was abrogated upon LILRB2 blockade. M-CSF monocyte-derived macrophages matured in the presence of LILRB2 antagonism showed increased sensitivity to LPS and IFN- $\gamma$ stimuli as indicated by enhanced STAT1, ERK, and NF- $\mathrm{B}$ phosphorylation, but were resistant to IL-4 stimulation as shown by reduced STAT6 phosphorylation and DC-SIGN expression along with increased SOCS3. Interestingly, LILRB2 blockade itself augmented NF- $\mathrm{KB}$ and ERK1/2 phosphorylation and decreased AKT phosphorylation, implying that LILRB2 antagonism alone is sufficient to moderately drive M-CSF monocyte-derived macrophages toward an M1 lineage. Nevertheless, LILRB2 antagonism suppressed an M2-associated and IL-4driven gene cluster while promoting those associated with an M1 phenotype. We proposed an LILRB2-mediated signal regulation model (Figure 7F) in which LILRB2 blockade can suppress SHP1/2 phosphorylation and lead to an M1 program through restored activation of ERK (53) and p38 by suppression of SHP1/DUSPmediated direct or indirect dephosphorylation (ERK at Y204 and p38 at Y182), leading to further activation of NF- $\kappa B$. Consistent with literature (54), downregulation of SHP1/SHP2 recruitment/ activation led to removal of a suppressor of JAK/STAT1 signaling and thus enhanced the IFN-induced signaling cascade (55). On the other hand, LILRB2 antagonism inhibited STAT6 phosphorylation by increasing SOCS3, which has been reported to suppress IL-4/STAT6 signaling (56). The induction of SOCS3 could result from the anti-LILRB2-mediated release of proinflammatory cytokines (IL-6 and IL-12) through activation of MAPK/NF- $\mathrm{BB}$ cascades $(57,58)$. Phosphorylation of AKT was significantly inhibited by LILRB2 blockade, which could be due to potential inhibition of (a) $\operatorname{SOCS} 3(59,60)$ or (b) PI3K $\gamma$ or SHIP-1 - two molecules that have been identified as key regulators for the macrophage switch between immune stimulation and suppression $(61,62)$.

We have previously shown that adoptive transfer of Pirbdeficient MDSCs decreases tumor burden and lung metastases. Modulating macrophage function represents an attractive approach to cancer immunotherapy since a body of literature has shown that TAMs are often a major component of the tumor microenvironment that contributes to tumor immune evasion (2). TAM-targeted therapeutics focusing on macrophage depletion highlight the clinical benefit in reducing tumor burden in mouse models (63). However, CSF1R inhibitors can enhance circulating levels of protumor granulocytic MDSCs, while withdrawal of CCL2 neutralization treatment can accelerate monocyte rebound to tumors 
A

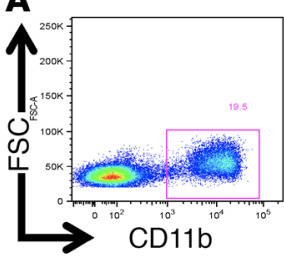

B

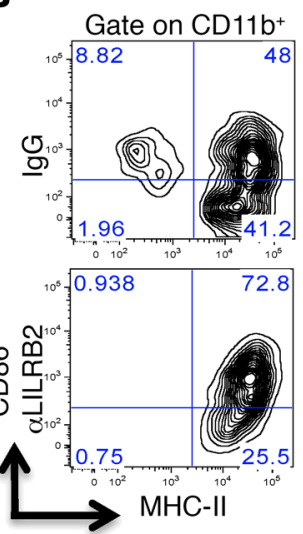

LIL R R B

E
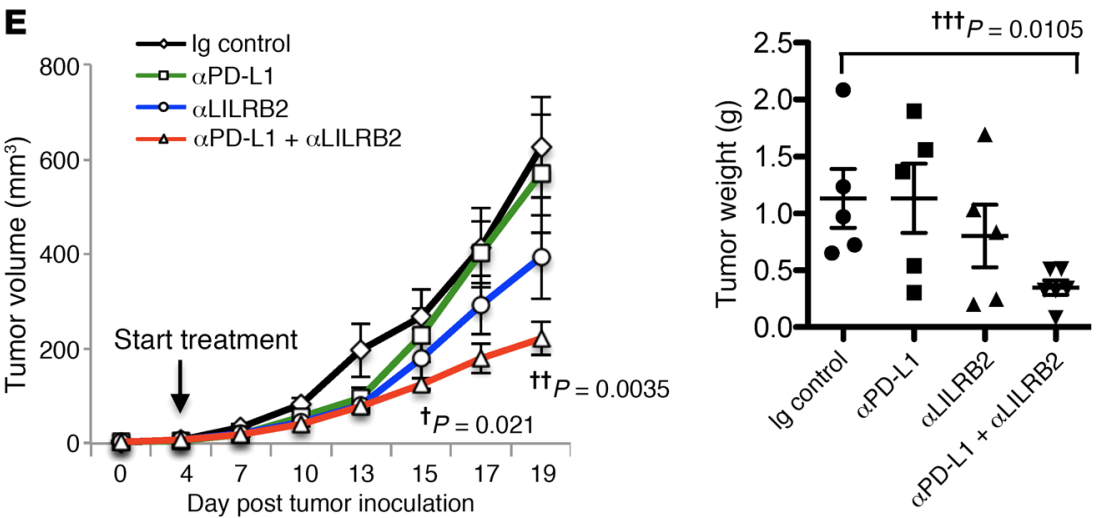

$\mathbf{F}$
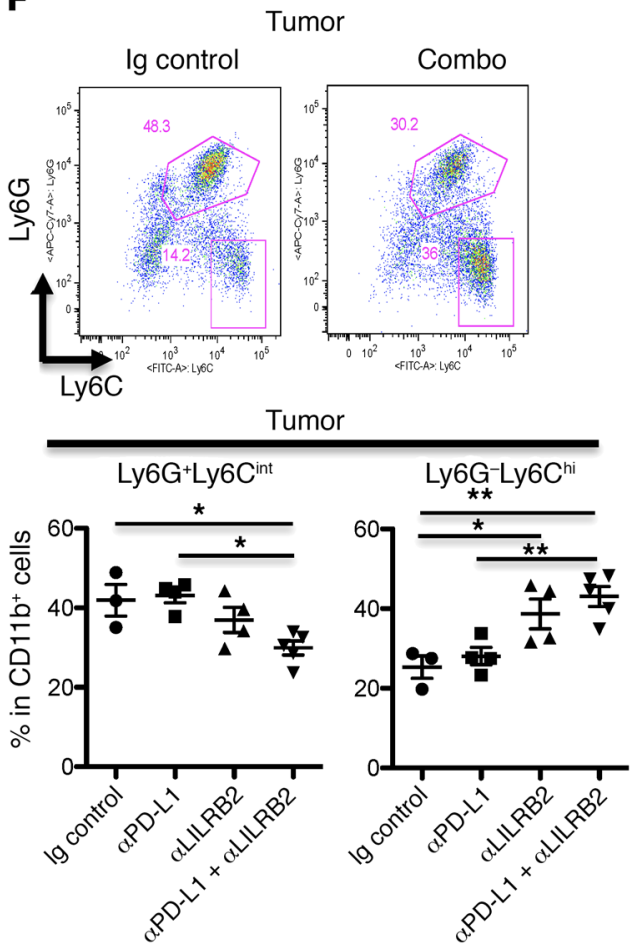

C

$O \lg G$

- $\alpha$ LILRB2
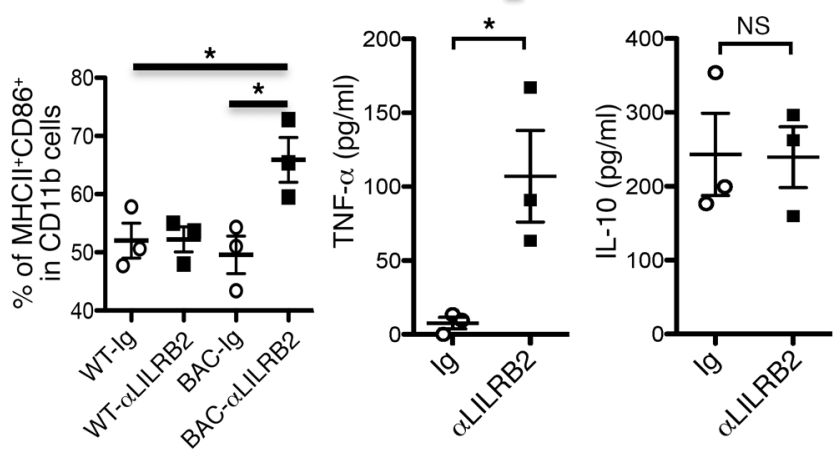

Figure 6. Antitumor effect of LILRB2 blockade in LILRB2-transgenic mice in LLC tumor model.

(A) LILRB2 and LILRB3 expression in CD11b-gated viable peripheral blood cells. (B) Total peripheral blood cells were harvested for flow cytometric analysis after LPS stimulation in the presence of IgC or anti-LILRB2 ( $\alpha$ LILRB2), and supernatants were collected for ELISA assay. Representative dot plots of the CD86 ${ }^{+} \mathrm{MHC}-\mathrm{II}^{+}$population in CD11b-gated cells. (C) Statistical analysis of percentage of CD86+MHC-II+ cells in CD11b-gated cells from $\mathbf{B}(n=3)$.(D) Statistical analysis of TNF- $\alpha$ secretion from total peripheral blood cells $(n=3)$. (E) Lewis lung carcinoma (LLC) tumor model in LILRB2-transgenic mice. Experimental mice were treated with Ig control or anti-LILRB2 ( $\alpha$ LILRB2) and/or anti-PD-L1 ( $\alpha$ PD-L1) . Left: LLC tumor growth was measured every 2 or 3 days and the average tumor volume per group \pm SEM reported $(n=5-6) .{ }^{\dagger} P=0.021,{ }^{\dagger \dagger} P=0.0035$ and, ${ }^{t+t} P=0.0105$ by 2 -way ANOVA comparison of groups on day 23. (F) Tumor-infiltrating lymphocytes were purified for flow cytometric analysis of Ly6C+Ly6C int granulocytic MDSCs and Ly6C-Ly6C hi monocytic MDSCs in the CD11 b+ cell population. Top: Representative flow cytometric analysis of MDSCs from tumors of test mice treated with Ig control or anti-LILRB2 plus anti-PD-L1. Bottom: Pooled MFI data from the flow cytometric plots above collected from tumor-bearing mice $(n=3-5$ mice per group. ${ }^{*} P<0.05$ and ${ }^{* *} P<0.01$, by 1 -way ANOVA followed by Tukey's multiple-comparisons test. (G) Splenocytes and tumor-infiltrating lymphocytes were isolated for flow cytometric analysis of $\mathrm{CD}_{25} 5^{+} \mathrm{FoxP3}^{+}$Tregs in $\mathrm{CD} 4^{+}$cell population. Top: Representative flow cytometric analysis of Tregs from spleens and tumors of the test mice treated with Ig control or anti-LILRB2 plus anti-PD-L1. Bottom: Pooled MFI data collected from the same tumor-bearing mice ( $n=4-6$ mice per group. ${ }^{*} P<0.05$, by 1 -way ANOVA followed by Tukey's multiple-comparisons test. 
$(64,65)$. Consequently, enhanced metastases and worse outcome are observed in several mouse models of breast cancer upon treatment cessation. TAMs and M2-like macrophages appear to share functional similarities (66). Immunotherapies that reprogram TAMs to support adaptive antitumor immunity offer an alternative strategy that avoids the potential pitfalls associated with TAM depletion (67). LILRB2 blockade appeared to alter the tumor-dependent maturation of macrophages similarly to what was observed with monocyte-derived macrophage cultures. Furthermore, we demonstrated enhanced acute TNF- $\alpha$ release and increased phagocytic capabilities in response to LILRB2 antibody treatment in xenograft humanized mice. Patient biopsies from NSCLC showed significant infiltration of MDSC and TAM populations. These primary MDSCs/ TAMs expressed high levels of LILRB proteins and were capable of undergoing the macrophage phenotype switch in the presence of LILRB2 blockade in ex vivo studies. LILRB2 appeared to be a critical negative regulator in both primary monocyte-derived macrophages and TAM populations harvested from patients. We also observed that anti-LILRB2 significantly decreased $\mathrm{Ly}^{6} \mathrm{G}^{+}$ Ly6C ${ }^{\text {int }}$ granulocytic MDSCs in both spleen and tumor tissues in an immune-competent syngeneic LILRB-transgenic mouse model.

Most importantly, LILRB2 antagonism substantially enhanced the efficacy of an immune checkpoint inhibitor, anti-PD-1, in a PBMC humanized NCG mouse model bearing human lung cancer cells, A549 (Figure 5E). We further confirmed the effect of LILRB2 blockade in an immunocompetent syngeneic Lilrb2/3 BAC transgenic mouse model (Figure 6E). It is important to note that anti-PD-L1 treatment alone showed a limited antitumor effect in LLC tumor-bearing immune-competent mice $(68,69)$. We found that LILRB2 antagonism suppressed LPS-induced CD274 (PDL1) expression in activated monocytes from healthy donors (Figure 2) and that blockade of both LILRB2 and PD-L1 potentiated a strong host antitumor immunity against LLC tumors in LILRB2transgenic mice. Concomitantly, we observed decreases in granulocytic MDSCs and Tregs in tumor-bearing mice treated with anti-LILRB2 and anti-PD-L1. The unexpected increase in monocytic MDSCs did not compromise the efficacy of anti-PD-L1, suggesting that LILRB2 antagonism converted monocytic MDSCs into a nonsuppressive and immunostimulatory phenotype, similar to that of Pirb-deficient MDSCs (12) and macrophages in the presence of LILRB2 blockade (Figure 5).

Several pathways have been shown to be involved in M2 polarization, including $\mathrm{mTOR} / \mathrm{semaphorin}$ 6D (Sema6D)/peroxisome proliferator receptor $\gamma$ (PPAR $\gamma$ ) (70), PI3K $\gamma / \mathrm{mTOR}(62)$, and TSC/mTOR pathways $(71,72)$. Targeting these pathways can potentially provide high therapeutic value in immune oncology. Our transcriptome data indicated that LILRB2 blockade markedly downregulated multiple gene targets involved in M2-like maturation, while genes associated with enhanced adaptive immunity and costimulation were upregulated. Our study highlighted that antagonism of LILRB2 inhibited both AKT activation and IL-4 signaling, which may interfere with Sema6D/PI3K $\gamma / \mathrm{mTOR}$ signaling pathways. This suggests that antagonism of LILRB2 may have broader impacts on reversing the immunosuppressive function of TAMs to enhance the efficacy of immune checkpoint inhibitors. Our data provide strong evidence that LILRB2 antagonism could be a promising approach for reprogramming
TAMs in the tumor microenvironment, thereby enhancing adaptive antitumor immunity.

\section{Methods}

Animals. NSG-SGM3 (stock 013062) and MISTRG mice (stock 017712) were purchased from The Jackson Laboratory. NCG mice (stock 572) were purchased from Charles River Laboratories. All animal experiments were conducted in accordance with the animal guidelines of the Icahn School of Medicine at Mount Sinai and the Houston Methodist Research Institute.

Cell lines. 2B4 reporter cells expressing Lilrb1-Lilrb4 and Lilra1 were generated by transfection with plasmids carrying corresponding Lilr-Fc fusion genes followed by selection by genome selection/marker-assisted selection (GS/MAS) systems and maintained in DMEM (Corning Cellgro) containing 10\% FBS (Atlantic Biologicals) plus 100 nM penicillin/streptomycin (Life Technologies). THP1 cells, a promonocytic cell line, were cultured in RPMI 1640 (Corning Cellgro) supplemented with $10 \%$ FBS, $0.1 \mathrm{mM}$ nonessential amino acids (Gibco), $1 \mathrm{mM}$ sodium pyruvate. THP1 and human alveolar adenocarcinoma A549 cell lines were purchased from the American Type Culture Collection. LILRB2 ${ }^{+}$THP-1 cells were generated by retroviral transfection with full-length LILRB2 plasmid.

Flow cytometric analysis and generation of anti-LILRB2 hybridoma. Commercial anti-LILRB2 antibodies, clone 42D1 and clone 287219, were purchased from BioLegend and eBioscience, respectively. Anti-LILRB1 (clone GHI/75), anti-LILRB3 (clone MKT5.1), and anti-LILRB4 (clone ZM4.1) antibodies were from BioLegend. Anti-LILRA1 (clone 586326) antibody was from Novus Biologicals. Human anti-CD4-FITC (clone RPA-T4), anti-CD8-PE (clone HIT8a), antiCD16-FITC (clone CB16), anti-DC-SIGN-PE (clone eB-h209), antiCD163-APC (clone GHI/61), anti-PD-L1-PerCP-Cy5.5 (clone MIH1), anti-CD33-PE-Cy7 (clone WM-53), anti-CD14-APC-Cy7 (clone 61D3), anti-CD45-PE (clone 2D1), and anti-HLA-DR-FITC (clone LN3) were purchased from eBioscience or BioLegend. Mouse anti-CD11b-PerCP (clone M1/70), anti-CD8a-eFluor 780 (clone 53-6.7), anti-CD4-FoxP3 (clone FJK-16S), anti-CD4-FITC (clone GK1.5), anti-CD25-PE-Cy7 (clone PC61.5), anti-MHC-II-PE-Cy7 (clone M5/114.15.2), anti-Ly6GAPC-Cy7 (clone 1A8), and anti-CD25-PE-Cy7 (clone PC61.5) were purchased from eBioscience or BioLegend. Anti-Ly6C-FITC (clone AL-21) and anti-mouse arginase 1-APC (clone IC5868F) were purchased from BD Biosciences and R\&D Systems, respectively.

We generated anti-LILRB2 antibodies by immunizing with Lilrb2 DNA followed by boosting once with LILRB2 vesicle or protein. Outgrowing fused hybridoma clones were expanded in vitro, and supernatants were screened using FACS on 2B4 LILRB2-expressing cells labeled with goat polyclonal anti-mouse IgG secondary antibody (BioLegend). The 2B4 cells were provided by Hisashi Arase (Research Institute for Microbial Diseases, Osaka University).

For functional screening of anti-LILRB2 antibodies, PBMCs were incubated with $20 \mu \mathrm{l}$ of antibody-containing supernatants for 48 hours. LPS (50 ng/ml; Sigma-Aldrich) and GolgiPlug (BD Biosciences) were added for the last 4 hours of cell culture. The cells were harvested for surface CD86 (anti-CD86-Alexa Fluor 488, clone IT2.2, BioLegend) and intracellular TNF- $\alpha$ (anti-TNF- $\alpha-\mathrm{PE}-\mathrm{Cy} 7$, clone MAB11, BioLegend) per the manufacturer's instructions (GolgiPlug Kit, BD Biosciences). In flow cytometric analyses, dead cells were excluded by DAPI (Sigma-Aldrich) staining. 
A

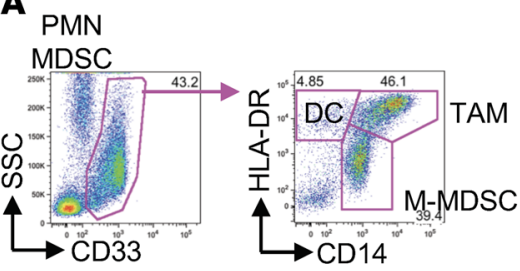

C

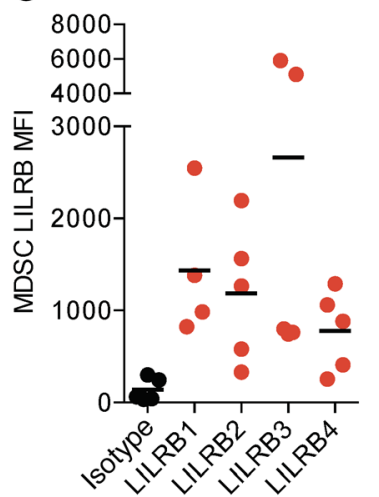

B

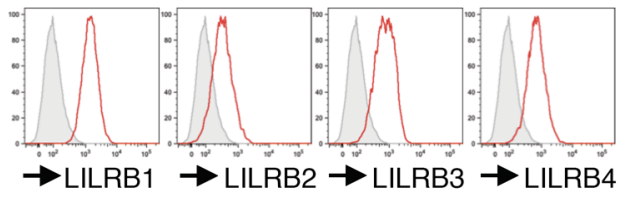

D

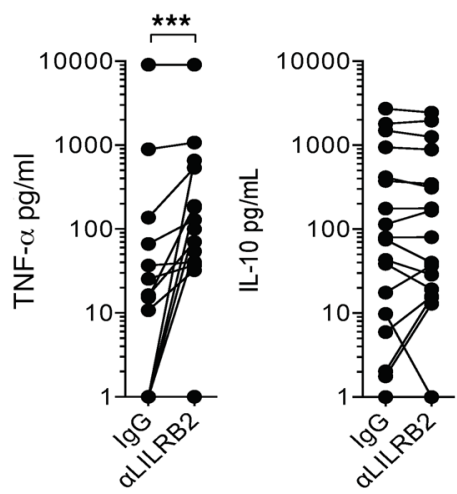

Figure 7. Tumor-infiltrating myeloid cells derived from lung cancer and mesothelioma patients respond to LILRB2 blockade. (A) Characterization of myeloid cell populations isolated from NSCLC excised tumor. Cells were gated on DAPI-CD45 ${ }^{+}$live leukocyte cells. PMN-MDSC, M-MDSC, DC, and TAM populations were identified. (B) Representative LILRB1-LILRB4 staining among $\mathrm{CD}_{3} 3^{+}$gate from $\mathbf{A}$. (C) LILRB MFI of multiple patient biopsies $(n=5-6)$ shown gated on the MDSC (left) and TAM (right) gates. (D) Lung cancer- and mesothelioma-derived infiltrating lymphocytes (TILs) were cultured with M-CSF plus IFN- $\gamma$ with Ig controls or anti-LILRB2 ( $\alpha$ LILRB2) for 48 hours. The supernatants were evaluated for TNF- $\alpha$ and IL-10 secretion $(n=15) .{ }^{* *} P<$ 0.001 , by paired $t$ test. (E) Lung cancer- and mesothelioma-derived TILs from D were collected from multiple patients for flow cytometric analysis for expression of CD163, CD14, and PD-L1 $(n=11)$ as well as DC-SICN and CD16 $(n=7) .{ }^{*} P<0.05$, by paired $t$ test. (F) Proposed mechanisms for the effect of LILRB2 antagonism on M1/M2 polarization.
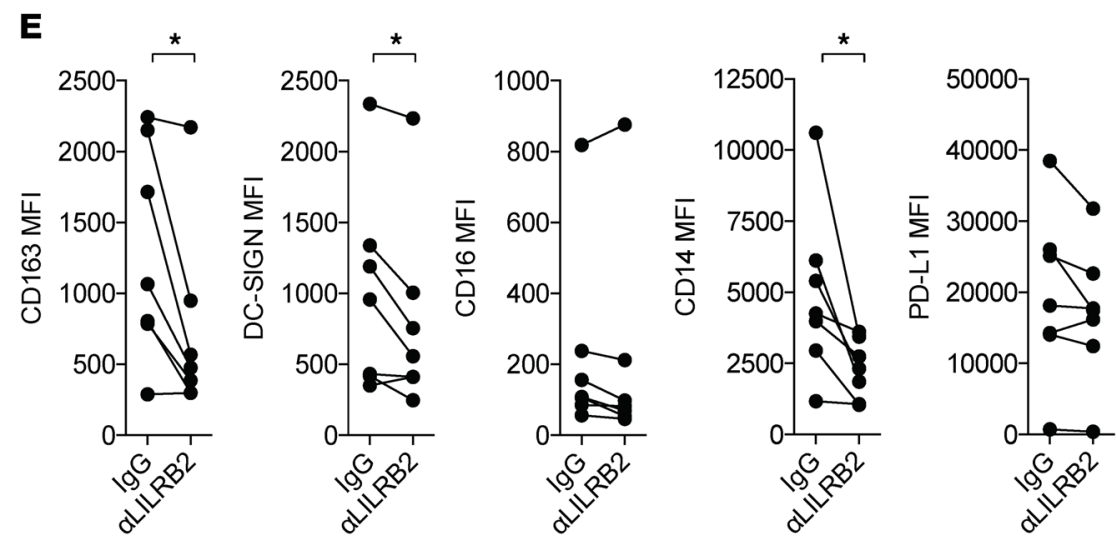

$\mathbf{F}$

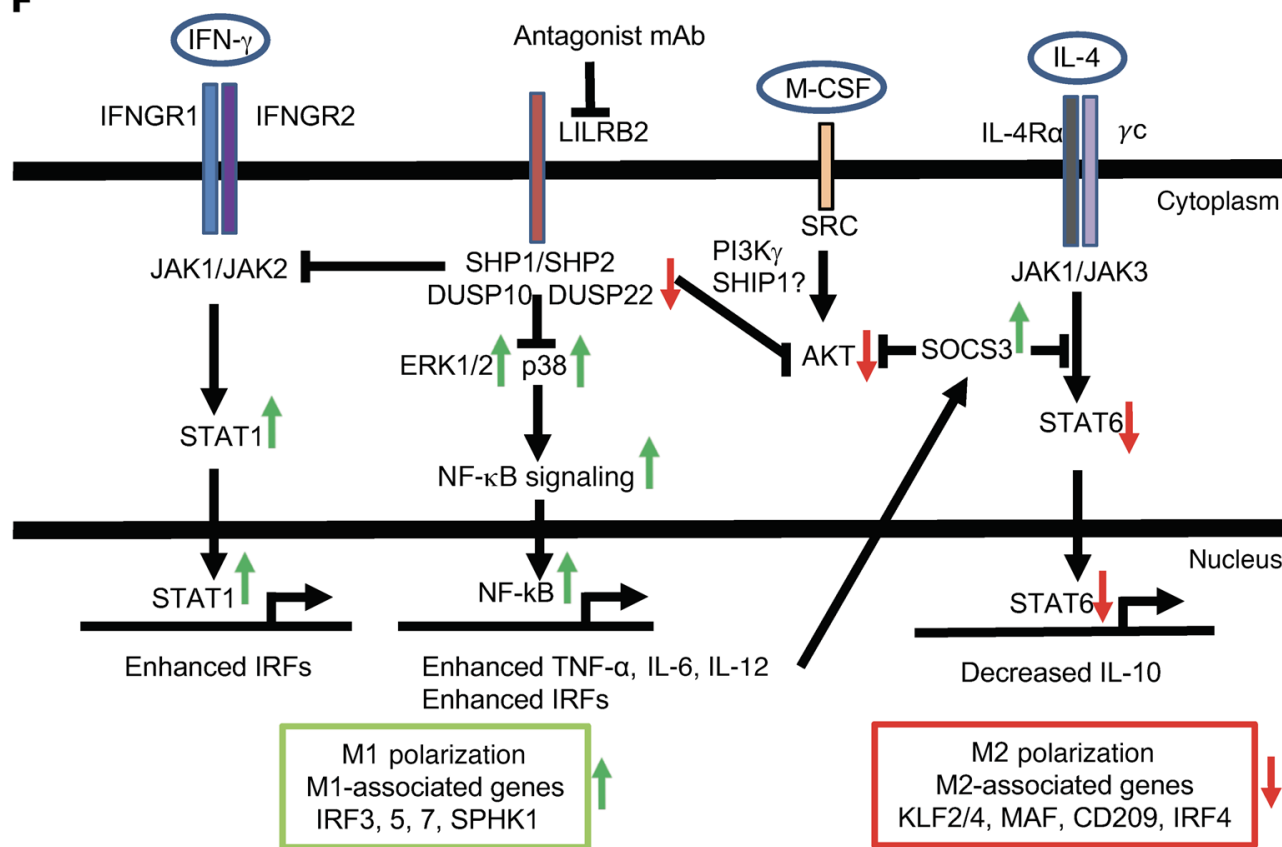


Biolayer interferometry binding assay. Real-time binding assays between LILRB2-His (Sino Biologicals) and anti-LILRB2 antibodies were performed using biolayer interferometry on an Octet Red system (FortéBio). This system monitors interference of light reflected from the surface of a fiber optic sensor to measure the thickness of molecules bound to the sensor surface. Anti-LILRB2 antibody $(10 \mu \mathrm{g} / \mathrm{ml})$ was coupled to kinetics-grade protein $\mathrm{G} /$ mouse IgG high-binding biosensors (FortéBio). Sensors coated with anti-LILRB2 antibody were allowed to bind to LILRB2-His in PBS with $0.1 \%$ (vol/vol) Tween-20 and 10\% DMSO at increasing concentrations. Binding kinetics were calculated using the Octet Red software package, which determined the best fit for the observed binding curves and calculated the association rate constants. LILRB2-His was dissociated by incubation of the sensors in PBS with 0.1\% Tween-20 and $10 \%$ DMSO. Best-fit dissociation curves were determined, and the dissociation rate constants were calculated. Binding affinities $\left(K_{\mathrm{D}}\right)$ were calculated as the kinetic dissociation rate constant divided by the kinetic association rate constant.

Antibody purification and endotoxin test. Clonal hybridoma cells were cultured in ClonaCell-HY Medium A (STEMCELL Technologies) followed by adaptation to serum-free conditions using Hybridoma-SFM medium (Thermo Fisher Scientific). Hybridoma cells were expanded in $50 \mathrm{ml}$ of Hybridoma-SFM for 2 weeks or until medium was exhausted. Antibody-containing supernatant was harvested by centrifugation (800 $g, 10$ minutes) and concentrated using Amicon Ultra-15 centrifugal filter concentrators with a nominal molecular weight limit of $100 \mathrm{kDa}$. Concentrated supernatants were then purified using Nab Protein A/G Spin Kit (Thermo Fisher Scientific) according to the manufacturer's instructions. Purified antibody was desalted using Zeba Spin Desalting Columns, 7K MWCO (Thermo Fisher Scientific). Purified antibodies were further concentrated by centrifugal filter (Thermo Fisher Scientific) if needed. Endotoxin levels in purified antibodies were determined by Pierce LAL Chromogenic Endotoxin Quantitation Kit (Thermo Fisher Scientific) according to the manufacturer's protocol.

Generation and differentiation of human monocyte-derived macrophages. Buffy coats from healthy donors were purchased from the New York Blood Center. PBMCs were isolated using LymphoPrep medium (07851, STEMCELL Technologies). CD33 ${ }^{+}$monocytes were purified from healthy $\mathrm{PBMC}$ donors using $\mathrm{CD}_{3} 3^{+}$magnetic beads (catalog 130-045-501, Miltenyi Biotec) and treated with IgG or anti-LILRB2 $(1 \mu \mathrm{g} / \mathrm{ml})$ in the presence of M-CSF (50 ng/ml) (300-25, PeproTech) for 5 days. After 5 -day culture, immature macrophages were obtained for flow analysis or further stimulated with LPS (50 ng/ml) or IL-4 (25 $\mathrm{ng} / \mathrm{ml}$ ) for 16-24 hours. The supernatants were collected, and TNF- $\alpha$ and IL-10 production was determined by ELISA (eBioscience).

A549 xenograft model in NSG-SGM3 immunodeficient mice. $3 \times 10^{6}$ A549 cells and $\mathrm{CD}_{3}{ }^{+}$myeloid cells from healthy donors were suspended in 50\% Matrigel (catalog 356231, Corning) and coinoculated s.c. into NSG-SGM3 mice (013062, The Jackson Laboratory). Each mouse received 2 subcutaneous A549/CD33 ${ }^{+}$implants in the right and left flank (day 0). Anti-RB2 antibody and corresponding Ig controls were injected i.v. on day 6 and day $9(150 \mu \mathrm{g} / \mathrm{mice})$. The tumors were measured and dissected for analysis on day 12. Tumor-infiltrating lymphocytes were purified as previously described for flow cytometric analysis of M1/M2 differentiation.

Luciferase-expressing A549 xenograft model in HLA-A2 $2^{+}$PBMC humanized NCG mice. Human luciferase-expressing A549 cancer cells
(LUC-A549; GenTarget Inc.) $\left(2 \times 10^{6}\right.$ cells $)$ were injected i.v. into NCG immunodeficient mice on day 0. Human HLA-A2 ${ }^{+}$PBMCs $\left(1 \times 10^{7}\right.$ cells $)$ were purified and injected i.v. into test mice together with anti-LILRB2 on day 3 and day 13. Anti-LILRB2 and IgG (200 $\mu \mathrm{g} /$ mice) were given once every 3 days for a total of 8 injections. Anti-PD-1 (Nivolumab) was given once per week ( $200 \mu \mathrm{g} /$ mice $)$ for a total of 3 injections. Subsequently, the LUC-A549 tumor-bearing mice were monitored for bioluminescent imaging of luciferase activity using the IVIS Spectrum In Vivo Imaging System and Living Image software (PerkinElmer Inc.). Before imaging, $250 \mu \mathrm{l}$ luciferin (at $15 \mathrm{mg} / \mathrm{ml}$; PerkinElmer) was administered intraperitoneally. Afterward, the mice were anesthetized by isoflurane inhalation and imaged. Tumor progression was quantified by bioluminescence signals (average radiance, $\mathrm{p} / \mathrm{s} / \mathrm{cm}^{2} / \mathrm{sr}$ ) from the lungs 25 days after tumor inoculation.

Generation of BAC transgenic mice. Bacterial artificial chromosome (BAC) clones for both the human Lilrb2 and Lilrb3 genes were isolated from a human BAC library (BACPAK Resources Center). BAC DNA was purified by NucleoBond Xtra Midi EF Kit. The identity of BAC DNA was confirmed via PCR for our gene of interest (Lilrb2), then microinjected into embryonic stem cells. Germline transmission of the targeted allele was confirmed by PCR and flow cytometry.

Lewis lung carcinoma tumor model in Lilrb2-transgenic mice. Lewis lung carcinoma (LLC) tumor cells $\left(4 \times 10^{5}\right.$ cells) were injected s.c. into LILRB2 B6 transgenic mice. Antibody treatments were started when tumors reached $2 \mathrm{~mm} \times 2 \mathrm{~mm}$. Anti-LILRB2 $(200 \mu \mathrm{g} / \mathrm{mouse})$ treatment was started on day 4 after tumor inoculation for a total of 6 injections. AntiPD-L1 (200 $\mu \mathrm{g} /$ mouse) treatment was started with the second injection of anti-LILRB2 for a total of 5 injections. The tumors were dissected for analysis on day 21. Splenocytes and tumor-infiltrating lymphocytes were purified for flow cytometric analysis of MDSC and Treg populations.

Phagocytosis of GFP-expressing E. coli ex vivo. Humanized MISTRG mice were screened by assessment of human $\mathrm{CD} 33^{+}$cell engraftment and equally distributed between human IgG control and anti-LILRB2 groups. The test mice were injected i.p. with anti-LILRB2 Ab $(150 \mu \mathrm{g} /$ mouse) for 48 hours. Peripheral blood cells were isolated and incubated with E. coli expressing GFP at the ratio of $2 \times 10^{8}$ E. coli per $1 \times 10^{7}$ peripheral blood cells for 4 hours at $37^{\circ} \mathrm{C}$. Duplicates per mouse were performed. After the incubation, the cells were washed with PBS and analyzed by gating on viable $\mathrm{CD} 45^{+} \mathrm{CD} 33^{+} \mathrm{CD} 14^{+}$population.

CpG challenge experiment. MISTRG newborns received a low dose of radiation (150 rad), then were intrahepatically injected with $5 \times 10^{4} \mathrm{CD}^{2} 4^{+}$human stem cells from cord blood (catalog 70008, STEMCELL Technologies) as previously described (73). After 8 weeks, the mice were checked for engraftment of $\mathrm{CD} 45^{+}$and $\mathrm{CD} 33^{+}$ population in the peripheral blood. The naive humanized MISTRG mice were injected i.v. with IgG control or anti-LILRB2 antibodies ( $150 \mu \mathrm{g} / \mathrm{mouse})$ for 2 days, and then challenged i.p. with $5 \mathrm{nmol} \mathrm{CpG}$ (catalog ODN1668, Invivogen). After 2 hours, serum was collected and analyzed using ELISA for TNF- $\alpha$ levels.

Western blot and coimmunoprecipitation. LILRB2-transduced THP1 cells were treated with $1 \mu \mathrm{g} / \mathrm{ml}$ IgG or anti-LILRB2 for 24 hours followed by acute stimulation with $50 \mathrm{ng} / \mathrm{ml} \mathrm{LPS}, 20 \mathrm{ng} / \mathrm{ml} \mathrm{IFN-} \gamma$, or $20 \mathrm{ng} / \mathrm{ml} \mathrm{IL}-4$ for 5, 10, and 30 minutes. The cells were lysed using cell lysis reagent (Sigma-Aldrich). Protein samples were separated on $8 \%$ SDS-polyacrylamide gels and transferred to PVDF membranes. The membranes were blocked in $4 \%$ skim milk solution, incubated with an appropriate antibody, and subsequently incubated with a second- 
ary antibody conjugated to horseradish peroxidase. The antibodies for p-STAT1, p-ERK1/2, p-p38, p-NF-кB p65, p-STAT6, SOCS1, SOCS3, p-AKT, SHP1, and p-SHP1 were purchased from Cell Signaling Technology, and the antibody for actin was purchased from Santa Cruz Biotechnology. The immunoreactive bands were visualized with the ECL system (Thermo Fisher Scientific). For immunoprecipitation, LILRB2 ${ }^{+}$THP1 cells were treated with IgG or anti-LILRB2 for 24 hours and $0.1 \mathrm{mM} \mathrm{Na}_{3} \mathrm{VO}_{4}$ (Sigma-Aldrich) for the last hour. The cells were lysed using cell lysis buffer including $1 \mathrm{mM} \mathrm{Na}_{3} \mathrm{VO}_{4}$ and $25 \mathrm{mM} \alpha$-glycerophosphate. Dynabeads Protein G (Life Technologies) was used for pull-down. The pull-down samples were subjected to immunoblot assay and probed by anti-LILRB2, anti-SHP1, and anti-p-SHP1.

Human mixed lymphocyte reaction assay. Mature DCs for use as stimulator cells were generated by culturing of sorted $\mathrm{CD} 14^{+}$monocytes in the presence of human GM-CSF ( $50 \mathrm{ng} / \mathrm{ml}$; PeproTech) for 5 days followed by LPS stimulation. IgG-or anti-LILRB2-treated mature macrophages were generated as previously described. Allogeneic T cells as responder cells were purified from unrelated healthy donors and cocultured with mature DCs and titrated ratios of macrophages for 72 hours (Supplemental Figure 2A). No antibody was present in the culture for the duration of the mixed lymphocyte reaction (MLR). Cells and supernatants from MLRs were analyzed for CD4 ${ }^{+}$and CD8 ${ }^{+}$ T cell number by FACS and IFN- $\gamma$ secretion by ELISA.

Transcriptome analysis. IgG- or anti-LILRB2-treated monocytederived macrophages from 3 healthy donors were subjected to microarray analysis. RNA was hybridized to Human HT-12 v4 Expression BeadChips (Illumina), and Illumina HiScan was used for scanning. Raw intensity data were processed using GenomeStudio (version 2011.1) Gene Expression Module (version 1.9.0; Illumina) and further processed using the lumi R package from Bioconductor. The data were adjusted for background signal before export from BeadStudio (Illumina) and underwent VST transformation and quantile normalization. Probes with no expression were removed. Differential gene expression analysis was performed using the limma $\mathrm{R}$ package, and significantly differentially expressed genes were identified based on a fold change of $\geq 1.5$ and a $P$ value less than 0.05 with Benjamini-Hochberg FDR correction. Heatmaps of differentially expressed genes were created using the plots R package, and unsupervised hierarchical clustering was performed based on Euclidean distance. Expression values were $Z$ score-normalized, and high and low expression is shown as red and green, respectively, with intermediate expression as white. Principal component analysis was performed using the prcomp function in R. The accession number for the microarray data is GSE117340 (Gene Expression Omnibus database)

Reverse transcriptase PCR. Reverse transcriptase PCR (RT-PCR) was performed on total RNA prepared by the TRIzol Reagent method. Two micrograms of RNA was used for cDNA synthesis using M-MLV reverse transcriptase (Promega) and oligo(dT) 18 (Thermo Fisher Scientific). Indicated below are the sequences of $5^{\prime}$ and $3^{\prime}$ primers used for each of the tested genes: IRF3: forward 5'-AGGTCCACAGTATTCTCCAGG-3', reverse 5'-AGGTCCACAGTATTCTCCAGG 3'; IRF4: forward 5'-GCTGATCGACCAGATCGACAG 3', reverse 5'-CGGTTGTAGTCCTGCTTGC 3'; IRF5: forward 5'-GGGCTTCAATGGGTCAACG 3', reverse 5'-GCCTTCGGTGTATTTCCCTG 3'; IRF7: forward 5'-CCCAGCAGGTAGCATTCCC 3', reverse 5'-GCAGCAGTTCCTCCGTGTAG 3'.

Isolation of infiltrating leukocytes from human lung cancer tissue. Eleven fresh lung cancer samples, including NSCLC and mesotheli- oma, were obtained from the Lung and Esophageal Surgery Institute, Mount Sinai Medical Center. Tumor samples were digested with $2 \mathrm{mg} /$ $\mathrm{ml}$ Collagenase D (Sigma-Aldrich) for $30-45$ minutes at $37^{\circ} \mathrm{C}$. The tissue lysates were then filtered through a $40-\mu \mathrm{m}$ cell strainer and the flow-through washed with PBS at $400 \mathrm{~g}$ for 5 minutes twice to pellet tumor cells. Lung cancer- or mesothelioma-derived infiltrating lymphocytes (TILs) were isolated by density gradient medium (LymphoPrep, catalog 07801, STEMCELL Technologies). TILs (100,000 to 200,000) were cultured with $50 \mathrm{ng} / \mathrm{ml} \mathrm{M-CSF}$ (PeproTech) in the presence or absence of $50 \mathrm{ng} / \mathrm{ml} \mathrm{IFN- \gamma}$ (PeproTech), LPS (SigmaAldrich), and IgG or anti-LILRB2 at $1 \mu \mathrm{g} / \mathrm{ml}$ for 2 days. Cells and supernatants were subjected to flow cytometric analysis and TNF- $\alpha / \mathrm{IL}-10$ production by ELISA, respectively.

Statistics. All data are presented as the means \pm SD. Data were compared using 2-tailed Student's $t$ test or 1-way ANOVA followed by Tukey's multiple-comparisons test. Paired $t$ test was used to compare results from the same treatments from different healthy donors or cancer patients. All analyses were conducted using Prism (GraphPad Software) and SPSS (IBM). Data were considered statistically significant at a $P$ value less than 0.05 .

Study approval. All animal procedures were approved by the IACUC at the Center for Comparative Medicine and Surgery of the Icahn School of Medicine at Mount Sinai and at Comparative Medicine at Houston Methodist Research Institute. The portion of this study involving human tissues was approved by the Institutional Review Board of the Icahn School of Medicine at Mount Sinai and was conducted in accordance with federal and institutional guidelines.

\section{Author contributions}

$\mathrm{SR}$ and RF procured clinical lung cancer specimens under IRB protocols. $\mathrm{RF}$ assisted with humanized mouse experiments in the MISTRG model. WVDT, HMC, and SHC wrote the manuscript with edits by PYP, RF, RF, SR, and SA." to "SR and R. Flores procured clinical lung cancer specimens under IRB protocols. R. Flavell assisted with humanized mouse experiments in the MISTRG model. WVDT, HMC, and SHC wrote the manuscript with edits by PYP, R. Flores, R. Flavell, SR, and SA.

\section{Acknowledgments}

We thank Marc Feldman, Ramon Parsons, and Cheng Cheng Zhang for the constructive discussion. We also thank Guangjie $\mathrm{Yu}$, Zhang Yao, and Yunong Pang for their technical support. This work was supported by National Cancer Institute (NCI) grants and Methodist Research funds to SHC and PYP. This work was supported in part by grants from the NCI to SHC (R01-CA109322, R01CA127483, and R01-CA208703, and U54-CA210181 Center for Immunotherapeutic Transport Oncophysics [CITO] pilot grant) and to PYP (R01-CA140243, R01-CA188610, and U54-CA210181 CITO pilot grant), and to SA (U01 OH011328-02). This work was also supported by NCI training grants T32-CA078207 (to WVDT and DAB), F32-AI122715 (to WVDT), and T32-GM062754 (to SM).

Address correspondence to: Ping-Ying Pan or Shu-Hsia Chen, Immunotherapy Research Center, Houston Methodist Research Institute, 6670 Bertner Avenue, Houston, Texas 77030, USA. Email:ppan@houstonmethodist.org (PYP).Email:shuhsia.chen@ houstonmethodist.org (SHC). 
1. Ortega-Gómez A, Perretti M, Soehnlein O. Resolution of inflammation: an integrated view. EMBO Mol Med. 2013;5(5):661-674.

2. Noy R, Pollard JW. Tumor-associated macrophages: from mechanisms to therapy. Immunity. 2014;41(1):49-61.

3. Martinez FO, Gordon S. The M1 and M2 paradigm of macrophage activation: time for reassessment. F1000Prime Rep. 2014;6:13.

4. van der Touw W, Chen HM, Pan PY, Chen SH. LILRB receptor-mediated regulation of myeloid cell maturation and function. Cancer Immunol Immunother. 2017;66(8):1079-1087.

5. Zhang J, et al. Leukocyte immunoglobulin-like receptors in human diseases: an overview of their distribution, function, and potential application for immunotherapies. J Leukoc Biol. 2017;102(2):351-360.

6. Ujike A, Takeda K, Nakamura A, Ebihara S, Akiyama $\mathrm{K}$, Takai T. Impaired dendritic cell maturation and increased $\mathrm{T}(\mathrm{H}) 2$ responses in PIR-B (-/-) mice. Nat Immunol. 2002;3(6):542-548.

7. Munitz A, et al. Paired immunoglobulin-like receptor $\mathrm{B}$ (PIR-B) negatively regulates macrophage activation in experimental colitis. Gastroenterology. 2010;139(2):530-541.

8. Endo S, Sakamoto Y, Kobayashi E, Nakamura A, Takai T. Regulation of cytotoxic $\mathrm{T}$ lymphocyte triggering by PIR-B on dendritic cells. Proc Natl Acad Sci U S A. 2008;105(38):14515-14520.

9. Takai T. Paired immunoglobulin-like receptors and their MHC class I recognition. Immunology. 2005;115(4):433-440.

10. Ho LH, Uehara T, Chen CC, Kubagawa H, Cooper MD. Constitutive tyrosine phosphorylation of the inhibitory paired Ig-like receptor PIR-B. Proc Natl Acad Sci U S A. 1999;96(26):15086-15090.

11. Maeda A, Kurosaki M, Ono M, Takai T, Kurosaki T. Requirement of SH2-containing protein tyrosine phosphatases SHP-1 and SHP-2 for paired immunoglobulin-like receptor $B$ (PIR-B)-mediated inhibitory signal. J Exp Med. 1998;187(8):1355-1360.

12. Ma G, et al. Paired immunoglobin-like receptor-B regulates the suppressive function and fate of myeloid-derived suppressor cells. Immunity. 2011;34(3):385-395.

13. Ravetch JV, Lanier LL. Immune inhibitory receptors. Science. 2000;290(5489):84-89.

14. Bashirova AA, Apps R, Vince N, Mochalova Y, $\mathrm{Yu}$ XG, Carrington M. Diversity of the human LILRB3/A6 locus encoding a myeloid inhibitory and activating receptor pair. Immunogenetics. 2014;66(1):1-8.

15. Slukvin II, Grendell RL, Rao DS, Hughes AL, Golos TG. Cloning of rhesus monkey LILRs. Tissue Antigens. 2006;67(4):331-337.

16. Mori Y, et al. Inhibitory immunoglobulin-like receptors LILRB and PIR-B negatively regulate osteoclast development. JImmunol. 2008;181(7):4742-4751.

17. Colonna M, et al. Human myelomonocytic cells express an inhibitory receptor for classical and nonclassical MHC class I molecules. J Immunol. 1998;160(7):3096-3100.

18. Cheng H, et al. Crystal structure of leukocyte Ig-like receptor LILRB4 (ILT3/LIR-5/ CD85k): a myeloid inhibitory receptor involved in immune tolerance. J Biol Chem. 2011;286(20):18013-18025.

19. Zhang Z, et al. The leukocyte immunoglobulinlike receptor family member LILRB 5 binds to HLA-class I heavy chains. PLoS One. 2015;10(6):e0129063.

20. Jones DC, et al. HLA class I allelic sequence and conformation regulate leukocyte Ig-like receptor binding. JImmunol. 2011;186(5):2990-2997.

21. Zheng J, et al. Inhibitory receptors bind ANGPTLs and support blood stem cells and leukaemia development. Nature. 2012;485(7400):656-660.

22. Shiroishi M, et al. Human inhibitory receptors Ig-like transcript 2 (ILT2) and ILT4 compete with CD8 for MHC class I binding and bind preferentially to HLA-G. Proc Natl Acad Sci U S A. 2003;100(15):8856-8861.

23. Lichterfeld $\mathrm{M}$, et al. A viral CTL escape mutation leading to immunoglobulin-like transcript 4-mediated functional inhibition of myelomonocytic cells. J Exp Med. 2007;204(12):2813-2824.

24. Shakhawat A, Shaikly V, Elzatma E, Mavrakos E, Jabeen A, Fernández N. Interaction between HLA-G and monocyte/macrophages in human pregnancy. J Reprod Immunol. 2010;85(1):40-46.

25. Banchereau J, et al. Immunoglobulin-like transcript receptors on human dermal CD14+ dendritic cells act as a CD8-antagonist to control cytotoxic T cell priming. Proc Natl Acad Sci U S A. 2012;109(46):18885-18890.

26. Chang CC, et al. Tolerization of dendritic cells by $\mathrm{T}(\mathrm{S})$ cells: the crucial role of inhibitory receptors ILT3 and ILT4. Nat Immunol. 2002;3(3):237-243.

27. Martinez FO, Gordon S, Locati M, Mantovani A. Transcriptional profiling of the human monocyte-to-macrophage differentiation and polarization: new molecules and patterns of gene expression. J Immunol. 2006;177(10):7303-7311.

28. Murray PJ, et al. Macrophage activation and polarization: nomenclature and experimental guidelines. Immunity. 2014;41(1):14-20.

29. Hashimoto S, Yamada M, Motoyoshi K, Akagawa KS. Enhancement of macrophage colony-stimulating factor-induced growth and differentiation of human monocytes by interleukin-10. Blood.1997;89(1):315-321.

30. Hashimoto S, Suzuki T, Dong HY, Yamazaki N, Matsushima K. Serial analysis of gene expression in human monocytes and macrophages. Blood. 1999;94(3):837-844.

31. Ino $\mathrm{Y}$, et al. Immune cell infiltration as an indicator of the immune microenvironment of pancreatic cancer. Br J Cancer. 2013;108(4):914-923.

32. Zaki MA, et al. Prognostic implication of types of tumor-associated macrophages in Hodgkin lymphoma. Virchows Arch. 2011;459(4):361-366.

33. Etzerodt A, Moestrup SK. CD163 and inflammation: biological, diagnostic, and therapeutic aspects. Antioxid Redox Signal. 2013;18(17):2352-2363.

34. Krausgruber T, et al. IRF5 promotes inflammatory macrophage polarization and TH1-TH17 responses. Nat Immunol. 2011;12(3):231-238.

35. Zhao GN, Jiang DS, Li H. Interferon regulatory factors: at the crossroads of immunity, metabolism, and disease. Biochim Biophys Acta. 2015;1852(2):365-378.
36. Negishi $\mathrm{H}$, et al. Negative regulation of Toll-like-receptor signaling by IRF-4. Proc Natl Acad Sci U S A. 2005;102(44):15989-15994.

37. Fleetwood AJ, Dinh H, Cook AD, Hertzog PJ, Hamilton JA. GM-CSF- and M-CSF-dependent macrophage phenotypes display differential dependence on type I interferon signaling. J Leukoc Biol. 2009;86(2):411-421.

38. Tanaka T, Murakami K, Bando Y, Yoshida S. Interferon regulatory factor 7 participates in the M1-like microglial polarization switch. Glia. 2015;63(4):595-610.

39. Rodríguez-García M, et al. Expression of PD-L1 and PD-L2 on human macrophages is up-regulated by HIV- 1 and differentially modulated by IL-10. JLeukoc Biol. 2011;89(4):507-515.

40. Conde P, et al. DC-SIGN (+) macrophages control the induction of transplantation tolerance. Immunity. 2015;42(6):1143-1158.

41. Domínguez-Soto A, et al. Dendritic cell-specific ICAM-3-grabbing nonintegrin expression on M2-polarized and tumor-associated macrophages is macrophage-CSF dependent and enhanced by tumor-derived IL- 6 and IL-10. JImmunol. 2011;186(4):2192-2200.

42. Smith JL, et al. ets-2 is a target for an Akt (protein kinase B)/jun N-terminal kinase signaling pathway in macrophages of motheaten-viable mutant mice. Mol Cell Biol. 2000;20(21):8026-8034.

43. Kelley TW, et al. Macrophage colony-stimulating factor promotes cell survival through Akt/protein kinase B. J Biol Chem. 1999;274(37):26393-26398.

44. Arranz A, et al. Akt1 and Akt2 protein kinases differentially contribute to macrophage polarization. Proc Natl Acad Sci US A. 2012;109(24):9517-9522.

45. Eden E, Navon R, Steinfeld I, Lipson D, Yakhini Z. GOrilla: a tool for discovery and visualization of enriched GO terms in ranked gene lists. BMC Bioinformatics. 2009;10:48.

46. Eden E, Lipson D, Yogev S, Yakhini Z. Discovering motifs in ranked lists of DNA sequences. PLoS Comput Biol. 2007;3(3):e39.

47. de las Casas-Engel M, et al. Serotonin skews human macrophage polarization through HTR2B and HTR7. J Immunol. 2013;190(5):2301-2310.

48. Gabriel TL, et al. Induction of Sphk1 activity in obese adipose tissue macrophages promotes survival. PLoS One. 2017;12(7):e0182075.

49. Kang K, et al. Interferon- $\gamma$ represses M2 gene expression in human macrophages by disassembling enhancers bound by the transcription factor MAF. Immunity. 2017;47(2):235-250.e4.

50. Tanoue T, Moriguchi T, Nishida E. Molecular cloning and characterization of a novel dual specificity phosphatase, MKP-5. J Biol Chem. 1999;274(28):19949-19956.

51. Li JP, et al. The phosphatase JKAP/DUSP22 inhibits T-cell receptor signalling and autoimmunity by inactivating Lck. Nat Commun. 2014;5:3618.

52. Chomarat P, Banchereau J, Davoust J, Palucka AK. IL-6 switches the differentiation of monocytes from dendritic cells to macrophages. Nat Immunol. 2000;1(6):510-514.

53. Nakata K, Suzuki Y, Inoue T, Ra C, Yakura H, Mizuno K. Deficiency of SHP1 leads to sustained and increased ERK activation in mast cells, thereby inhibiting IL-3-dependent proliferation and cell 
death. Mol Immunol. 2011;48(4):472-480.

54 . Blanchette J, Abu-Dayyeh I, Hassani K, Whitcombe L, Olivier M. Regulation of macrophage nitric oxide production by the protein tyrosine phosphatase Src homology 2 domain phosphotyrosine phosphatase 1 (SHP-1). Immunology. 2009;127(1):123-133.

55. Böhmer FD, Friedrich K. Protein tyrosine phosphatases as wardens of STAT signaling. JAKSTAT. 2014;3(1):e28087.

56. Hebenstreit D, Luft P, Schmiedlechner A, Duschl A, Horejs-Hoeck J. SOCS-1 and SOCS-3 inhibit IL-4 and IL-13 induced activation of Eotaxin-3/CCL26 gene expression in HEK293 cells. Mol Immunol. 2005;42(3):295-303.

57. Carow B, Rottenberg ME. SOCS3, a major regulator of infection and inflammation. Front Immunol. 2014;5:58.

58. White CA, Nicola NA. SOCS3: an essential physiological inhibitor of signaling by interleukin-6 and G-CSF family cytokines. JAKSTAT. 2013;2(4):e25045.

59. Zhang L, et al. IL-6 and serum amyloid A synergy mediates angiotensin II-induced muscle wasting. JAm Soc Nephrol. 2009;20(3):604-612.
60. Gordon P, Okai B, Hoare JI, Erwig LP, Wilson HM. SOCS3 is a modulator of human macrophage phagocytosis. JLeukoc Biol. 2016;100(4):771-780.

61. Rauh MJ, et al. The role of SHIP1 in macrophage programming and activation. Biochem Soc Trans. 2004;32(pt 5):785-788.

62. Kaneda MM, et al. PI3K $\gamma$ is a molecular switch that controls immune suppression. Nature. 2016;539(7629):437-442.

63. Ries $\mathrm{CH}$, et al. Targeting tumor-associated macrophages with anti-CSF-1R antibody reveals a strategy for cancer therapy. Cancer Cell. 2014;25(6):846-859.

64. Bonapace L, et al. Cessation of CCL2 inhibition accelerates breast cancer metastasis by promoting angiogenesis. Nature. 2014;515(7525):130-133.

65. Swierczak A, et al. The promotion of breast cancer metastasis caused by inhibition of CSF-1R/CSF-1 signaling is blocked by targeting the G-CSF receptor. Cancer Immunol Res. 2014;2(8):765-776.

66. Mantovani A, Sozzani S, Locati M, Allavena P, Sica A. Macrophage polarization: tumorassociated macrophages as a paradigm for polarized M2 mononuclear phagocytes. Trends Immunol. 2002;23(11):549-555.

67. Mantovani A, Allavena P. The interaction of anticancer therapies with tumor-associated macrophages. JExp Med. 2015;212(4):435-445.

68. Li HY, et al. The tumor microenvironment regulates sensitivity of murine lung tumors to PD-1/ PD-L1 antibody blockade. Cancer Immunol Res. 2017;5(9):767-777.

69. Lin H, et al. Host expression of PD-L1 determines efficacy of PD-L1 pathway blockade-mediated tumor regression. J Clin Invest. 2018;128(4):1708.

70. Kang S, et al. Semaphorin 6D reverse signaling controls macrophage lipid metabolism and anti-inflammatory polarization. Nat Immunol. 2018;19(6):561-570.

71. Wilson JL, Weichhart T. TORching a semaphore for alternative macrophage activation. Nat Immunol. 2018;19(6):512-514.

72. Byles V, et al. The TSC-mTOR pathway regulates macrophage polarization. Nat Commun. 2013;4:2834.

73. Rongvaux A, et al. Development and function of human innate immune cells in a humanized mouse model. Nat Biotechnol. 2014;32(4):364-372. 